\title{
Novel mediation analysis of human plasma proteome and metabolome reveals mediators of improved glycemia after gastric bypass surgery
}

Jonathan M Dreyfuss ${ }^{1,2^{\star}}$, Yixing Yuchi ${ }^{3,4^{\star}}$, Hui Pan ${ }^{1,2}$, Xuehong Dong ${ }^{3,4}$, Donald C. Simonson ${ }^{4,5}$, Ashley Vernon ${ }^{4,6}$, Pratik Aryal ${ }^{4,7}$, Anish Konkar ${ }^{8,9}$, Yinong Sebastian ${ }^{8}$, Brandon W Higgs ${ }^{8}$, Joseph Grimsby ${ }^{8}$, Cristina M. Rondinone ${ }^{8}$, Simon Kasif ${ }^{1}$, Barbara B. Kahn ${ }^{4,7}$, Kathleen Foster ${ }^{3}$, Allison Goldfine ${ }^{3,4,10}$, Mary-Elizabeth Patti ${ }^{3,4,{ }^{* \star}}$

1 Biomedical Engineering, Boston University, Boston, MA USA

2 Bioinformatics and Biostatistics Core, Research Division, Joslin Diabetes Center, Boston, MA USA

3 Integrative Physiology and Metabolism, Research Division, Joslin Diabetes Center, Boston, MA USA

4 Harvard Medical School, Boston, MA USA

5 Department of Medicine, Brigham and Women's Hospital, Boston, MA USA

6 Department of Surgery, Brigham and Women's Hospital, Boston, MA USA

7 Division of Endocrinology, Diabetes \& Metabolism, Department of Medicine, Beth Israel

Deaconess Medical Center, Boston, MA USA

8 Medlmmune, Gaithersburg, MD USA

9 Present address: Sanofi-Aventis Deutschland GmbH, Frankfurt, Germany

10 Present address: Novartis Institutes for BioMedical Research, Cambridge, MA USA

${ }^{*}$ co-first authors

** Lead contact: MaryElizabeth.Patti@joslin.harvard.edu

Key Words: bariatric surgery; type 2 diabetes; obesity; mediation; pathway analysis; multiomic integration

\begin{abstract}
Molecular mechanisms by which Roux-en-Y gastric bypass (RYGB) improves glycemic control and metabolism in type 2 diabetes (T2D) remain incompletely understood. In the SLIMM-T2D trial, participants with T2D were randomized to RYGB or nonsurgical management and their fasting plasma proteome and metabolome were analyzed for up to 3 years. To identify analytes that mediate improvement in outcomes, we developed a high-throughput mediation analysis method (Hitman), which is significantly more powerful than existing methods. Top-ranking analyte mediators of glycemia improvement were growth hormone receptor and prolylhydroxyproline, which were more significant than any clinical mediator, including BMI. Betaalanine and Histidine Metabolism (both including CNDP1) were top differentially regulated pathways, and Valine, Leucine and Isoleucine Degradation was also a top differentiallyregulated pathway and a top mediator of improvement in insulin resistance. The identified
\end{abstract}


analytes may serve as novel targets for T2D therapy. More broadly, Hitman can identify analyte mediators of outcomes in randomized trials for which high-throughput data are available.

\section{Introduction}

Bariatric surgery is a potent approach to manage obesity and related comorbidities, including type 2 diabetes (T2D) and its complications (1-3). Roux-en-Y gastric bypass (RYGB) has particularly powerful effects on glucose metabolism, resulting in remission of T2D in about 90\% of patients at 1 year and $45 \%$ at 5 years (4-10). Improved glycemic control occurs days after RYGB, before substantial weight loss, supporting that weight loss-independent mechanisms drive metabolic improvements (11-13). Moreover, recent observational studies indicate that bariatric surgery is associated with fewer microvascular and macrovascular complications of diabetes and reduced mortality (14-16). Identifying molecular mechanisms responsible for improved systemic metabolism could allow development of novel nonsurgical approaches for T2D treatment.

Improved postoperative glycemic control is linked to enhanced meal-related insulin secretion and increases in incretin hormones such as GLP-1 and PYY (17-19). However, preclinical studies indicate incretins are not required for beneficial effects of bariatric procedures (20-24). Other postoperative changes include reductions in branched chain amino acids (BCAA)(25-27) and aromatic amino acids (28), alterations in plasma bile acids and FXR signaling (29-31) and in other hormones (32). RYGB alters the microbiome community composition toward that of less obese individuals (33), with increased abundance of many amino acid fermenters (34). Yet, microbiome changes cannot fully explain clinical improvement (35). Thus, the primary molecular factor(s) contributing to improved metabolism and diabetes remission remain uncertain.

We assayed fasting plasma samples from the Surgery or Lifestyle with Intensive Medical Management in the Treatment of Type 2 Diabetes (SLIMM-T2D) clinical trial (clinicaltrials.gov:NCT01073020), in which 38 obese participants with T2D were randomized to RYGB ( $\mathrm{n}=19)$ or nonsurgical intensive diabetes weight management $(D W M ; n=19)$ and followed longitudinally for 3 years $(10,36)$. Groups were similar at baseline (pre-randomization). Greater clinical improvement was seen in RYGB than in DWM for multiple outcomes, including body weight reduction (assessed by BMI), glycemia (assessed by HbA1c), triglycerides, HDL cholesterol, systolic blood pressure, and reductions in the number of antidiabetic, antihypertensive, and lipid-lowering medications (Simonson et al., 2018). All RYGB participants achieved $10 \%$ weight loss before 3 months, whereas 37\% of DWM participants achieved 10\% weight loss before 3 months (36). To identify candidate molecules responsible for improvement in glucose metabolism after RYGB as compared with DWM, we developed novel tools and applied them to clinical, proteomic, and metabolomic data from these samples. 


\section{Results}

\section{Clinical}

Proteomic and metabolomic data at baseline, the 3 month time point, and 1 year were available from 35 of the 38 SLIMM-T2D participants. Metabolic characteristics of these 35 participants did not differ between groups at baseline (Table S1). The HbA1C and BMI of those that had proteomics or metabolomics per time point also did not differ from the SLIMM-T2D participants within their group at any time point (Figure S1). After 3 years, no DWM participants achieved study-defined glycemic goals ( $\mathrm{HbA} 1 \mathrm{c}<6.5 \%$ and fasting plasma glucose $<126 \mathrm{mg} / \mathrm{dL}$ ), whereas eight RYGB participants did, and seven of these eight participants were not receiving any antidiabetes medications (Simonson et al., 2018). The number of participants taking each diabetes medication class per arm at each time point is tabulated in Table S1.

\section{Proteome}

Fasting proteomics were profiled at baseline, the 3 month time point, and years 1, 2 and 3 in RYGB and DWM. At baseline (pre-randomization), there were no statistically significant proteins as assessed by false discovery rate $(F D R<0.15)$. For later, post-randomization time points, reduction in BMI was greater in RYGB, so groups were compared both with and without adjustment for each person's BMI change. Differences in the baseline-corrected proteome in RYGB vs. DWM (i.e. differences between groups in changes over time) emerged at the 3 month time point, with 14 significant proteins in the unadjusted analysis and 8 in the BMI-adjusted analysis. Proteins common to both analyses and downregulated in RYGB were: carnosine dipeptidase 1 (CNDP1, also known as Beta-Ala-His dipeptidase 1) and Fetuin-B (FETUB). Proteins in common upregulated in RYGB were: integrin-binding sialoprotein (IBSP), insulin-like growth factor binding protein (IGFBP2), endothelial cell-specific molecule 1 (ESM1), macrophage metalloelastase (MMP12), alpha-1-antichymotrypsin complex (SERPINA3), and CC motif chemokine 22 (CCL22).

Differences of baseline-corrected protein abundance in RYGB vs. DWM persisted at years 1, 2, and 3. The $\log _{2}$ fold change values in RYGB vs. DWM for the 19 proteins differentially abundant with fold-change magnitude at least 1.5 after baseline correction at any time point without BMI adjustment are depicted in the heatmap in Figure 1. Statistics for all comparisons in all analytes are presented in Table S2. Upregulation of IGFBP2 $(>50 \%, p<0.001$ at all time points in SOMAscan, Figure S2A) was confirmed by ELISA, which showed significant differences in baseline-corrected values $(p<0.05)$ at 12,24 , and 36 months (Figure S2B), and ELISA changes over time were significantly correlated to corresponding SOMAscan changes $\left(r=0.78, p<10^{-7}\right)$.

\section{Metabolome}

At baseline (pre-randomization), there were no significant differences in the fasting metabolome between groups. Significant differences in the baseline-corrected metabolome in RYGB vs. DWM emerged at the 3 month time point, with 96 metabolites changed in the unadjusted 
analysis and 74 in the BMI-adjusted analysis; 45 of the metabolites were common to both analyses.

Differences of baseline-corrected metabolite abundance in RYGB vs. DWM persisted at years 1, 2 , and 3. The $\log _{2}$ fold change values in RYGB vs. DWM for the 85 metabolites differentially abundant with fold-change magnitude at least 1.5 after baseline correction at any time point without BMI adjustment are depicted in the heatmap in Figure 2. The color range was defined to match Figure 1, with fold-change magnitudes above 3 ( $\mid \log _{2}$ fold change| $\left.>1.6\right)$ treated as fold-change magnitude of 3 , the limit of the color bar. However, we saw much larger foldchanges in the metabolome. For example, the BCAA-related metabolite 3-hydroxyisobutyrate was $88 \%$ lower (i.e. down by $>9$-fold) at the 3 month time point in RYGB than DWM, and it was also significant in the BMI-adjusted analysis. Statistics for all comparisons in all analytes are presented in Table S2. For the metabolites in Figure 2, we tested the correlation of their change from baseline at the 3 month time point to the corresponding changes in the proteins shown in Figure 1 to identify co-regulation. These correlations are provided in Table S2.

\section{Proteomic and metabolomic integrative pathway analysis}

We integrated proteomics and metabolomics for pathway analysis by creating a single, integrated dataset, and testing pathways composed of both proteins and metabolites from the Small Molecule Pathway Database (37). We identified differentially abundant pathways between groups without BMI adjustment at the 3 month time point. The 3 month time point showed robust improvements in glucose metabolism in both groups, yet preceded larger weight differences. There were 50 significant pathways.

Top-ranking pathways are presented in Figure 3A. The top-ranking pathway is Phospholipid Biosynthesis (Figure 3B), whose top analytes were choline (Figure S2C) and choline phosphate (Figure S2D), both reduced in RYGB relative to DWM. The second pathway was Valine, Leucine, and Isoleucine Degradation, whose top analytes were the valine catabolic intermediate 3-hydroxyisobutyrate acid (88\% lower at the 3 month time point in RYGB; Figure 4, middle row, right), the ketoacid 3-methyl-2-oxobutyrate (ketoisovalerate; Figure 4, middle row, second from right), and the BCAAs valine and leucine (Figure 4, top row), all with sustained reductions after RYGB. Similar reductions were observed for other ketoacids and downstream acylcarnitines (Figure 4). Propionylcarnitine, a C3 acylcarnitine product of valine and isoleucine metabolism (Figure 4, lower row) with a 50\% reduction in RYGB, was the top analyte of the related pathway Oxidation of Branched Chain Fatty Acids.

Beta-alanine Metabolism's and Histidine Metabolism's (Figure 5A) top analytes were CNDP1 and its enzymatic product histidine. Histidine was reduced after RYGB but not DWM (FDR 0.002 , Figure 5D), and its reduction was correlated with the reduction of CNDP1 ( $r=0.40$, $\mathrm{p}=0.02)$. CNDP1 was reduced by $43 \%$ after RYGB at the 3 month time point $\left(\mathrm{FDR}=1.6^{\star} 10^{-5}\right.$ ) and remained lower in RYGB at 36 months (Figure 5B). A previous study had been unable to validate SOMAscan CNDP1 measurements (38). ELISA-determined CNDP1 levels in our analysis showed little correlation with SOMAscan levels per time point, but changes within person over time showed stronger correlation $(r=0.21, p=0.055)$. Moreover, we confirmed 
significant reductions in CNDP1 after RYGB, demonstrating a 68\% decrease in baselinecorrected change for RYGB vs. DWM ( $p=0.004$ at the 3 month time point, Figure $5 \mathrm{C}$ ).

Retinol Metabolism's top analyte was retinol (vitamin A), whose baseline-corrected abundance decreased by $33 \%$ in RYGB vs. DWM at the 3 month time point $\left(F D R<10^{-4}\right.$ ), and remained nominally lower at 12 months $(p=0.02$, FDR=0.18), but reverted to baseline values in both groups at 36 months (Figure S3A). Retinol binding protein 4 (RBP4) tended to be decreased in RYGB (10\% lower at the 3 month time point, Figure S3B); these changes in RBP4 were significantly correlated to changes in retinol over all time points $(r=0.33, p=0.001)$. Given the role of RBP4 as a mediator of insulin resistance (39), we measured RBP4 and its partner transthyretin (TTR) in a random subset of 12 subjects by quantitative western blot at multiple time points. At the 3 month time point, RBP4 and TTR were numerically lower in RYGB vs. DWM, but differences did not reach statistical significance with this sample size (Figure S3C, S3D). However, RBP4 changes by western blot correlated significantly to changes by SOMAscan $(r=0.28, p=0.03)$, and changes in both RBP4 and TTR significantly correlated to each other $\left(r=0.63, p<10^{-5}\right)$ and to changes in retinol (both $r>0.6, p<10^{-4}$ ).

\section{High-throughput Mediation Analysis (Hitman)}

We sought to identify analytes and clinical measures at the 3 month time point that mediated RYGB's improvement in glycemia at 1 year. While there are numerous mediation methods, two previous comprehensive comparisons, including one for genome-wide data, concluded that the best balance between false positive rate and power was offered by the joint significance method $(40,41)$. This method was mathematically shown to control its false positive rate and to be more powerful than some of its peers (42). A drawback of the joint significance test is that it does not account for direction of effect of the mediator, so it could call as significant an "inconsistent" mediator (43). For example, RYGB (compared to DWM) decreases retinol at the 3 month time point and $\mathrm{HbA} 1 \mathrm{c}$ at 1 year. However, a decrease in retinol is associated with an increase in HbA1c (Figure 6, left). Thus, the direction of retinol's mediation is inconsistent with RYGBmediated improvement in HbA1c.

A second limitation of the joint significance and other mediation methods is that they are intended for testing only one or a few mediators. Thus they do not include powerful statistical methods for high-throughput data, such as linear regression modeling with empirical Bayesian modeling of an analyte's variance, which has been validated in multiple omics platforms, and is particularly powerful for small sample sizes (44).

To address these limitations, we developed a novel mediation method for high-throughput data, termed High-thhroughput Mediation Analysis (Hitman). We demonstrate mathematically that it controls its false positive rate (Text S1), and it accounts for the direction of effect, so it does not identify inconsistent mediators as significant. For example, Hitman does not identify retinol as a significant mediator $(p>0.9)$. Hitman applies empirical Bayesian linear modelling, so it has improved power in high-throughput settings (44). 
Using simulations that followed the work of Barfield et al (41), we compared Hitman against the joint significance test and against a popular mediation test that uses the potential outcome framework (45) but was not included in previous simulations. We found that Hitman controls its false positive rate, like the other methods, and is significantly more powerful. For example, when the true causal mediation effects are of a size termed "small" (40), the joint significance and the potential outcome mediation test identify significance in $<2.5 \%$ of simulations, whereas Hitman identifies $>6 \%$, which is significantly greater $\left(p<10^{-15}\right)$. When the true effect is of a size termed "medium" (40), the proportion of simulations identified as significant by the joint significance test and the potential outcome mediation test were $53.9 \%$ and $57.4 \%$, respectively, whereas Hitman identified $68 \%$, which is also significantly greater $\left(p<10^{-15}\right)$. These simulation results, simulation details, and a description of the Hitman algorithm are provided in Text S1.

Mediation methods can be misled by confounding variables, such as those that affect both the mediator and the outcome (46). To avoid confounding among measured mediators, some highthroughput mediation approaches decompose the measured analytes to identify latent, independent mediators $(47,48)$. However in biology many confounders may be unmeasured. For example surgery has multifaceted effects, so decomposing our measured analytes would not be sufficient to prevent the impact of confounders, so Hitman does not attempt to control for confounders. In biological datasets where all confounders and causal relationships between analytes cannot be controlled for, causal mediation analysis should be considered as exploratory (49).

\section{Proteomic, metabolomic, integrative pathway, and clinical mediation analysis}

We applied Hitman to identify analytes whose early change (baseline to the 3 month time point) mediates HbA1c improvement at one year (Table S2). The only significant analyte was growth hormone receptor (GHR; $\left.p=10^{-4}, F D R=0.12\right)$. The second-ranking analyte was CNDP1 $\left(p=10^{-3}\right)$. Both decreased $>30 \%$ at the 3 month time point after RYGB (Table S2) and are shown in Figure 6. Robust GHR mediation led us to hypothesize that reductions in plasma GHR reflected reduced tissue content or altered receptor shedding, and thus could be associated with tissuelevel growth hormone resistance. In the liver, GHR signaling typically increases secretion of the growth factor IGF1 $(50,51)$; however, IGF1 was not identified as a mediator by Hitman. Moreover, neither SOMAscan nor ELISA measures of IGF1 differed between arms at the 3 month time point (Figure S5), despite robust correlation between SOMAscan and ELISA changes $(r=0.35, p=0.003)$. Even with unchanged IGF1 levels, plasma growth hormone levels were $>6$-fold higher in baseline-corrected RYGB vs. DWM at 12 months (Figure S5), raising the possibility that tissue- or pathway-selective growth hormone resistance in post-RYGB participants could contribute to sustained improvements in glucose metabolism (52).

The top-ranking putative metabolite mediators were prolylhydroxyproline $\left(p<10^{-3}, F D R=0.38\right)$ and isovalerylcarnitine ( $\mathrm{p}<0.005$; Figure 4, Figure 6, Figure S4, Table S2), which increased by $100 \%$ and decreased by $47 \%$ at the 3 month time point, respectively.

As a comparison to Hitman, we applied the joint significance method to test mediation of proteins and metabolites whose change at the 3 month time point mediates HbA1c 
improvement at one year. We found similar top analytes, but with weaker significance, consistent with our simulations demonstrating that Hitman offers substantial power (Text S1). The top-ranking analytes identified by the joint significance method were GHR (FDR=0.26) and pro-hydroxy-pro (FDR=0.73).

We next asked whether early postoperative change (baseline to the 3 month time point) in 40 clinical markers mediated $\mathrm{HbA} 1 \mathrm{c}$ improvement at one year. The top-ranking early postoperative mediators identified by Hitman were 6 min walk test distance and BMI ( $p<0.03$, FDR=0.4; Table S2). Strikingly, analytes identified in the 3 month time point mediation analysis (GHR and prolylhydroxyproline) were more significant (by p-value and FDR) than any clinical markers at the 3 month time point, suggesting the top analytes' utility as potential clinical biomarkers.

We next sought to test mediation of our integrated pathways. There are several approaches that test pathway mediation $(48,49)$, but they are applied to the data itself, so they cannot take Hitman scores as input. However, the CAMERA pre-ranked procedure accepts our Hitman scores as input and accounts for correlation between genes (53). CAMERA tests pathway enrichment while accounting for correlation between genes. When we tested for metabolic pathways whose change at the 3 month time point mediate HbA1c improvement at 1 year, no pathways were significant. However, the top-ranking pathways were Beta-Alanine Metabolism (Figure 5) and Histidine Metabolism ( $p=0.006$; FDR=0.23).

Given that improvements in glycemic control after RYGB are related to changes in insulin sensitivity and/or insulin secretion, we applied Hitman to identify analytes whose change from baseline at the 3 month time point mediated insulin secretion and insulin sensitivity change from baseline at 1 year (Table S2), and followed this with integrative pathway mediation analysis.

Insulin secretion, defined as the change in insulin from 0 to 30 minutes during a mixed meal tolerance test, was improved in RYGB vs. DWM (means of changes: RYGB=37.4, DWM=0.808; $\mathrm{p}=0.001$ ). No single analytes were identified as mediators, but one pathway, Caffeine Metabolism, significantly mediated improved insulin secretion (FDR $<10^{-4}$ ). Similarly, insulin sensitivity, defined by reduction in HOMA-IR, also improved in RYGB vs. DWM at 1 year (means of changes: RYGB=-2.12, DWM=-0.102; $\mathrm{p}=0.02$ ). No individual proteins or metabolites were significant mediators of insulin sensitivity, but many top ranking analytes were BCAArelated metabolites. Consequently, pathway analysis identified Valine, Leucine and Isoleucine Degradation as a significant pathway mediator of insulin sensitivity (FDR $\left.<10^{-7}\right)$, together with 14 other pathways, primarily involved in lipid and amino acid metabolism (Table S2).

\section{Discussion}

We report findings from a clinical trial that randomized individuals with T2D to RYGB or medical management and measured high-throughput data serially over 3 years. This design allowed us to identify analytes and pathways with differential patterns of change and potential mediators of improved metabolism after RYGB, providing candidates for nonsurgical approaches to T2D. This is shown in a graphical overview in Figure S6. As expected, RYGB exerted greater weight 
loss and glycemic improvement, and observed protein and metabolite changes are more robust during periods of active weight loss, indicating partial weight dependence of metabolic effects. However, differential abundance of many analytes remained after BMI adjustment, indicating weight-independence. Moreover, the top analytes at the 3 month time point are more significant mediators of glycemia at 1 year than any clinical markers, including BMI, indicating that these analytes may have clinical significance as biomarkers of bariatric surgery or therapeutic targets. Our mediation analysis tool, Hitman, provides a new method to analyze studies that randomize a treatment and observe differences in outcomes; using measurements of analytes upstream of the outcome, putative mediators of the outcome can be identified.

One feature of Hitman that improves its power is that it accounts for the direction of mediation. Hitman finds that retinol's direction of mediation is inconsistent with mediation of improved glycemic control after RYGB. As seen in Figure 3, RYGB reduces retinol more than DWM, which is compatible with previous reports in bariatric surgery (54-56). However, within both treatment groups, greater retinol reduction was associated with increased HbA1c, indicating that the reduction in retinol is not a likely mediator of improved glycemia. Similarly, retinol binding protein 4 (RBP4) was numerically lower after RYGB, consistent with prior reports linking increased serum RBP4 levels with obesity and insulin resistance (57). Like retinol, RBP4 reduction was associated with increased $\mathrm{HbA} 1 \mathrm{c}$ within each of the treatment groups, so it was also penalized by Hitman (Table S2). Thus, these data highlight complex relationships between retinol, RBP4, and T2D phenotypes, but suggest that these changes are not likely to mediate improved glycemia after RYGB.

Several significant pathways identified were driven by reductions in CNDP1, a top-ranking putative protein mediator of HbA1c. CNDP1 is a secreted dipeptidase that hydrolyzes carnosine to $\beta$-alanine and L-histidine. CNDP1 was found to decline significantly 3 months after bariatric surgery (58). These relationships are consistent with a common CNDP1 genetic variant that enhances enzymatic activity, which is associated with loss of glycemic control in mice (59).

The significant protein putative mediator of improved glycemic control was GHR, which was decreased in plasma from post-RYGB participants. Interestingly, reduction in GHR expression was recently demonstrated in multiple tissues of post-RYGB rodents (60). Parallel reductions in GHR and increased growth hormone levels post-RYGB suggest the possibility that tissueselective growth hormone resistance could contribute to improvements in insulin sensitivity and reductions in hepatic glucose production, as observed in humans treated with a growth hormone antagonist (52).

The top putative metabolite mediator of HbA1c was prolylhydroxyproline. Prolylhydroxyproline is a marker of collagen degradation, and the increased levels in RYGB could be linked to increased bone turnover (61) consistently observed after bariatric surgery, including in the present cohort $(62,63)$. One possible mechanism for mediation is that prolylhydroxyproline can facilitate adipose-derived stromal vascular cells to differentiate into more metabolically active beige adipocytes (64). 
Valine, Leucine, and Isoleucine Degradation was a significant HOMA-IR pathway mediator and the most significantly changed pathway at 3 months between groups. There were robust postRYGB decreases in BCAA and multiple downstream catabolic intermediates, including C3 and C5 acylcarnitines, with isovalerylcarnitine as a putative causal metabolite mediator. Our results are consistent with prior findings of increased BCAA in insulin resistance $(65,66)$ and reduced BCAA in response to RYGB in nonrandomized studies (25-27). Lower BCAA levels post-RYGB may be a consequence of weight loss-linked improvements in insulin sensitivity or altered microbial metabolism (34), but may also contribute directly to improved insulin sensitivity (67). Our data reveal a marked $88 \%$ reduction in the valine catabolic intermediate 3hydroxyisobutyrate at the 3 month time point post-RYGB. This is particularly interesting since 3hydroxyisobutyrate can exit mitochondria and serve as a signaling molecule, promoting muscle lipid uptake and insulin resistance (68) and impaired mitochondrial OXPHOS activity (69).

We acknowledge that profiling semi-quantitative plasma metabolomics and proteomics, with emphasis on the secreted proteome, cannot fully define the pleiotropic effects of bariatric surgery, including both weight-dependent and weight-independent changes in complex interorgan communication, gut microbiome effects, or tissue-specific flux in metabolic pathways. Our study is innovative as we have developed and applied novel bioinformatics tools to identify clinical measures, analytes and pathways that mediate response to RYGB within a randomized clinical trial comparing RYGB vs. medical management in T2D. The analytes we have identified and validated can be modulated in future studies to determine whether they can be utilized for non-surgical control of glucose metabolism in T2D.

\section{Materials and Methods}

\section{Clinical study}

Participants were randomized to RYGB, performed using standard operative protocols, or DWM, conducted by a multidisciplinary team in groups of 10-15 via the Why WAIT (Weight Achievement and Intensive Treatment) program (70). Both RYGB and DWM groups returned to usual care following intervention, and annual follow-ups after one year were observational. The protocol was approved by the Partners Healthcare Institutional Review Board, and an independent data monitoring committee reviewed patient safety. Difference of outcomes between groups was analyzed with a t-test, and significance was defined as $p<0.05$.

\section{$\underline{\text { Metabolic assessments }}$}

Metabolic assessments were performed at baseline and repeated at the 3 month time point, which was defined by achieving $10 \%$ of initial body weight loss or 3 months, if $10 \%$ weight loss had not yet been achieved by this time point. This time point was defined to permit assessments at a similar level of weight loss in both groups. Assessments were also repeated in both groups at 12, 18, 24, and 36 months. Analysis of blood samples collected in the fasting state included $\mathrm{HbA1C}$, plasma glucose, and lipids (Quest Diagnostics). Aliquots were stored at $-80 \mathrm{C}$. 


\section{Proteomic profiling and validation}

Plasma proteome profiling was performed using the high-throughput DNA aptamer-based SOMAscan assay platform (SomaLogic, Inc.)(71). Abundance of 1129 proteins (enriched for extracellular proteins) was quantified as relative fluorescent units (RFU), normalized, calibrated, and $\log _{2}$-transformed. Samples were available from 38 participants at baseline and 38, 35, 25 and 23 participants at the $3,12,24$, and 36 month time points, respectively (Table S1).

Selected proteomic data were validated in a subset of fasting plasma samples using specific ELISA, including IGFBP2 (22-BP2HU-E01, ALPCO, NH), CNDP1 (F34010, LifeSpan Biosciences, WA), growth hormone (DGH00, R\&D Systems, MN), and total IGF-1 (DG100, R\&D Systems, MN). For RBP4 and TTR, plasma samples were assayed using quantitative western blotting using a polyclonal antibody to human RBP4 (Dako) and human TTR (Dako) with standard curves of purified human RBP4 (72) or TTR (Sigma) protein on each blot (57). Changes per individual over time were tested for positive correlation to corresponding SOMAscan changes with a one-sided test of Pearson correlation.

\section{Metabolomic profiling}

Plasma metabolomics were profiled using a commercial semi-quantitative mass spectrometrybased platform (Metabolon, Inc.)(73). Metabolites that had missing values in more than $85 \%$ of samples were filtered out, missing values were imputed with half of the minimum for each metabolite, and abundance values were $\log _{2}$-transformed. Samples were available from 38 participants at baseline and 36, 35 and 22 participants at 3, 12, and 36 months; due to cost, metabolomics were not profiled at 24 months.

\section{Differential abundance of proteomics, metabolomics, and pathways}

To test differential abundance of $\log _{2}$ normalized analytes between groups at baseline, we applied moderated t-tests with our $\mathrm{R}$ package ezlimma, which streamlines and extends the $\mathrm{R}$ package limma $(44,74)$. Limma applies linear regression modeling with empirical Bayesian methods to improve each analyte's variance estimation using analytes' shared systematic variance. At post-baseline time points, we calculated change in analyte abundance from baseline for each individual, and then applied moderated t-tests to test if these changes varied by group. We repeated these post-baseline analyses accounting for each person's BMI change. We similarly applied limma's roast method (75) for differentially abundant pathways via our ezlimma package. We considered Roast's "Mixed" statistics, which correspond to assessing the absolute value of an analyte's change, so that if some of a pathway's analytes are upregulated (e.g. proteins) and others are down-regulated (e.g. metabolites), these changes do not cancel out the pathway's effect. Significance for this and other omics analyses was defined as FDR<0.15. In plots of individual analytes with standard error of the mean (SEM), ordinary (i.e. unmoderated) SEM are displayed. 


\section{Mediation analysis}

We tested mediation of each clinical variable with the causal chain: group $\rightarrow$ clinical variable change $\rightarrow$ clinical outcome change. We tested each clinical variable's mediation by defining group as a binary variable representing RYGB or DWM per individual; clinical variable change as each clinical variable's change between baseline and the 3 month time point per individual; and clinical outcome change as the change in clinical outcome between baseline and 12 months per individual.

We tested mediation of analytes with the causal chain: group $\rightarrow$ analyte change $\rightarrow$ clinical outcome change. We tested each analyte's mediation by defining group as a binary variable representing RYGB or DWM per individual; analyte change as each analyte's change (on the $\log _{2}$ scale) between baseline and the 3 month time point per individual; and clinical outcome change as the change in clinical outcome between baseline and 12 months per individual. As a comparison to Hitman, we similarly tested mediation of analytes using the joint significance method and using the mediation package (45) in the R software. Simulation results were compared statistically with a two-sample t-test with pooled variance in the $\mathrm{R}$ software.

We tested these Hitman results against pathways containing proteins and metabolites from the Small Molecule Pathway Database (SMPDB)(37) with the CAMERA pre-ranked pathway analysis method (53) from the Limma package.

\section{Data and software availability}

SOMAscan and clinical data have been deposited at GEO:GSE122279. SOMAscan, metabolomics, and clinical data, and the R/Bioconductor (74) code to reproduce main results from them (including Table S2 and pathway results with links to the underlying pathway's analyte statistics) are available at https://github.com/jdreyf/slimm-t2d-omics. Our streamlined limma $\mathrm{R}$ package ezlimma is available at https://github.com/jdreyf/ezlimma. The Hitman package is available at https://github.com/jdreyf/Hitman.

\section{Figure and table legends}

Figure 1. Proteome changes, see also Figure S2 and Table S2. Heatmap of $\log _{2}(\mathrm{RYGB} / D W M)$ at all time points (post-baseline $\log _{2}$ abundance values are baselinecorrected) for proteins that are differentially abundant at any time point (FDR $<0.15$ and foldchange magnitude $>1.5$ ).

Figure 2. Metabolome changes, see also Table S2. Heatmap of $\log _{2}(R Y G B / D W M)$ at all time points (post-baseline $\log _{2}$ abundance values are baseline-corrected) for metabolites that are differentially abundant at any time point (FDR $<0.15$ and fold-change magnitude $>1.5$ ). $\log _{2}$ values outside the range -1.58 to 1.58 are shrunken toward zero to be in this range. 
Figure 3: Top differential pathways and Phospholipid Biosynthesis network. $(A)-\log _{10}(p-$ values) and false discovery rates (FDRs) of top pathways. (B) Nodes are colored by betweengroup z-score, whereas unmeasured nodes are colored dark gray. Connections are from Pathway Commons network.

Figure 4. Branched chain amino acids (BCAA) and downstream metabolites, see also Table S2. Arrows indicate enzymatic pathway relationships. Data were analyzed by moderated t-tests; post-baseline time points were analyzed using change from baseline. Data are reported as mean \pm SEM on the $\log 2$ scale. ${ }^{*} p<0.05,{ }^{* *} p<0.01,{ }^{* \star *} p<0.001$, and $\# p<0.0001$.

Figure 5. Beta-alanine metabolism, see also Table S2. (A) Network where nodes are colored by between-group z-score, whereas unmeasured nodes are colored gray. Connections are from Pathway Commons network. (B) $\log _{2}$ abundance of CNDP1 measured by SOMAscan. (C) CNDP1 plasma levels measured by ELISA. (D) $\log _{2}$ abundance of histidine measured by metabolomics. Data were analyzed by moderated t-tests; post-baseline time points were analyzed using change from baseline. Data in $B, C$, and $D$ are reported as mean $\pm S E M$. ${ }^{*} p<0.05,{ }^{* *} p<0.01,{ }^{* * *} p<0.001$, and $\# p<0.0001$.

Figure 6. Mediation of HbA1c change at 1 year, see also Figure S1, S5, S6, Table S1, Table S2, and Text S1. X-axis represents $\log _{2}$ abundance change from baseline at the 3 month time point, and $\mathrm{Y}$-axis represents $\mathrm{HbA} 1 \mathrm{c}$ change from baseline at 1 year. Retinol is an inconsistent mediator, whereas GHR (growth hormone receptor) and pro-hydroxy-pro (prolylhydroxyproline) are consistent mediators of surgery's effect.

\section{Supplemental Information Items}

Figure S1. HbA1c and BMI for SLIMM-T2D cohort and those that had omics per time point. (A) HbA1C and (B) BMI in both treatment arms for the SLIMM-T2D cohort ("Total") and those that had proteomics or metabolomics per time point ("Omics"). Data are reported as mean \pm SEM. Data were analyzed by t-tests. For HbA1c and BMI, no significant difference was found between the Total and Omics groups within RYGB or within DWM at any time point. However, differences between RYGB and DWM per time point were found: ${ }^{*} p<0.05,{ }^{*} p<0.01$, $\star * \star p<0.001$ and $\# p<0.0001$.

Figure S2. IGFBP2, choline, and choline phosphate levels. (A) IGFBP2 $\log _{2}$ abundance measured by SOMAscan. (B) IGFBP2 concentration measured by ELISA. (C-D) Choline and choline phosphate $\log _{2}$ abundance measured by metabolomics. Data were analyzed by moderated t-tests; post-baseline time points were baseline-corrected. Data are reported as mean \pm SEM: ${ }^{*} p<0.05,{ }^{* \star} p<0.01,{ }^{\star * \star} p<0.001$, and $\# p<0.0001$.

Figure S3. Retinol, RBP4, and TTR abundance. (A) $\log _{2}$ abundance of retinol measured by metabolomics. (B) $\log _{2}$ abundance of RBP4 measured by SOMAscan. (C) RBP4 and (D) TTR plasma levels measured by quantitative western blot. Data were analyzed by moderated t-tests; post-baseline time points were baseline-corrected. Data are reported as mean \pm SEM: * $p<0.05$, ${ }^{* *} \mathrm{p}<0.01,{ }^{* * *} \mathrm{p}<0.001$ and $\# \mathrm{p}<0.0001$. 
Figure S4. Abundance of prolylhydroxyproline over time. $\log _{2}$ abundance of the mediator prolylhydroxyproline measured by metabolomics. Data were analyzed by moderated t-tests; post-baseline time points were baseline-corrected. Data are reported as mean \pm SEM: ${ }^{*}<<0.05$, ${ }^{* *} p<0.01,{ }^{* * *} p<0.001$ and $\# p<0.0001$.

Figure S5. IGF-1 and GHR SOMAscan and ELISA levels. (A) $\log _{2}$ abundance of IGF-1, measured by SOMAscan. (B) Plasma levels of total IGF-1, measured by ELISA. (C) $\log _{2}$ abundance of GHR, measured by SOMAscan. (D) Plasma levels of growth hormone, measured by ELISA. Data were analyzed by moderated t-tests; post-baseline time points were baselinecorrected. Data are reported as mean \pm SEM: ${ }^{*} p<0.05,{ }^{* *} p<0.01,{ }^{* \star *} p<0.001$ and $\# p<0.0001$.

Figure S6. Graphical overview. Shows study flow. Bottom show top two mediators (GHR and prolyl-hydroxy-proline) and a network composed of top analytes from Valine, Leucine, and Isoleucine Degradation and Beta-Alanine Metabolism. Nodes colored dark grey were not measured here.

Table S1. Baseline demographics of participants that had both proteomics and metabolomics at all of baseline, 3 months, and 12 months; and number of participants taking each diabetes medication class per arm at each time point. The metabolic characteristics of the subset of participants who had omics data did not differ between groups at baseline. There were differences in medication use between groups.

Table S2. Differential abundance, correlation, and mediation tables. Differential abundance of proteins, metabolites, and pathways; Pearson correlation coefficients of top proteins vs. top metabolites; mediation of $\mathrm{HbA1c}$, insulin secretion (as the change in insulin from 0 to 30 minutes during a mixed meal tolerance test), and HOMA-IR.

Text S1. Hitman algorithm and validation. Technical description and validation.

\section{References}

1. R. O'Brien et al., Microvascular Outcomes in Patients With Diabetes After Bariatric Surgery Versus Usual Care: A Matched Cohort Study. Ann Intern Med 169, 300-310 (2018).

2. L. Sjostrom et al., Association of bariatric surgery with long-term remission of type 2 diabetes and with microvascular and macrovascular complications. JAMA 311, 22972304 (2014).

3. L. Sjostrom, Review of the key results from the Swedish Obese Subjects (SOS) trial - a prospective controlled intervention study of bariatric surgery. J Intern Med 273, 219-234 (2013).

4. H. Buchwald et al., Bariatric surgery: a systematic review and meta-analysis. JAMA 292, 1724-1737 (2004).

5. P. R. Schauer et al., Bariatric Surgery versus Intensive Medical Therapy for Diabetes - 5Year Outcomes. N Engl J Med 376, 641-651 (2017). 
6. T. Olbers et al., Laparoscopic Roux-en-Y gastric bypass in adolescents with severe obesity (AMOS): a prospective, 5-year, Swedish nationwide study. Lancet Diabetes Endocrinol 5, 174-183 (2017).

7. G. Mingrone et al., Bariatric-metabolic surgery versus conventional medical treatment in obese patients with type 2 diabetes: 5 year follow-up of an open-label, single-centre, randomised controlled trial. Lancet 386, 964-973 (2015).

8. A. P. Courcoulas et al., Three-Year Outcomes of Bariatric Surgery vs Lifestyle Intervention for Type 2 Diabetes Mellitus Treatment: A Randomized Clinical Trial. JAMA Surg 150, 931-940 (2015).

9. D. E. Cummings et al., Gastric bypass surgery vs intensive lifestyle and medical intervention for type 2 diabetes: the CROSSROADS randomised controlled trial. Diabetologia 59, 945-953 (2016).

10. D. C. Simonson, F. Halperin, K. Foster, A. Vernon, A. B. Goldfine, Clinical and PatientCentered Outcomes in Obese Patients With Type 2 Diabetes 3 Years After Randomization to Roux-en-Y Gastric Bypass Surgery Versus Intensive Lifestyle Management: The SLIMM-T2D Study. Diabetes Care 41, 670-679 (2018).

11. C. Guidone et al., Mechanisms of recovery from type 2 diabetes after malabsorptive bariatric surgery. Diabetes 55, 2025-2031 (2006).

12. N. B. Jorgensen et al., Acute and long-term effects of Roux-en-Y gastric bypass on glucose metabolism in subjects with Type 2 diabetes and normal glucose tolerance. Am J Physiol Endocrinol Metab 303, E122-131 (2012).

13. P. R. Schauer et al., Effect of laparoscopic Roux-en Y gastric bypass on type 2 diabetes mellitus. Ann.Surg. 238, 467-484 (2003).

14. D. Arterburn, Microvascular Outcomes in Patients With Diabetes After Bariatric Surgery. Ann Intern Med 170, 507-508 (2019).

15. D. P. Fisher et al., Association Between Bariatric Surgery and Macrovascular Disease Outcomes in Patients With Type 2 Diabetes and Severe Obesity. JAMA 320, 1570-1582 (2018).

16. A. Aminian et al., Association of Metabolic Surgery With Major Adverse Cardiovascular Outcomes in Patients With Type 2 Diabetes and Obesity. JAMA, (2019).

17. R. D. Ramracheya et al., PYY-Dependent Restoration of Impaired Insulin and Glucagon Secretion in Type 2 Diabetes following Roux-En-Y Gastric Bypass Surgery. Cell reports 15, 944-950 (2016).

18. M. Salehi, R. L. Prigeon, D. A. D'Alessio, Gastric bypass surgery enhances glucagon-like Peptide 1-stimulated postprandial insulin secretion in humans. Diabetes 60, 2308-2314 (2011).

19. K. T. Nguyen et al., Preserved Insulin Secretory Capacity and Weight Loss Are the Predominant Predictors of Glycemic Control in Patients With Type 2 Diabetes Randomized to Roux-en-Y Gastric Bypass. Diabetes 64, 3104-3110 (2015).

20. M. Mokadem, J. F. Zechner, R. F. Margolskee, D. J. Drucker, V. Aguirre, Effects of Roux-en-Y gastric bypass on energy and glucose homeostasis are preserved in two mouse models of functional glucagon-like peptide-1 deficiency. Mol Metab 3, 191-201 (2014).

21. J. Ye et al., GLP-1 receptor signaling is not required for reduced body weight after RYGB in rodents. Am J Physiol Regul Integr Comp Physiol 306, R352-362 (2014).

22. N. Saeidi et al., Reprogramming of intestinal glucose metabolism and glycemic control in rats after gastric bypass. Science 341, 406-410 (2013). 
23. R. Dutia et al., Temporal changes in bile acid levels and 12alpha-hydroxylation after Roux-en-Y gastric bypass surgery in type 2 diabetes. Int J Obes (Lond) 39, 806-813 (2015).

24. A. D. Miras, C. W. le Roux, Mechanisms underlying weight loss after bariatric surgery. Nat Rev Gastroenterol Hepatol 10, 575-584 (2013).

25. B. Laferrere et al., Differential metabolic impact of gastric bypass surgery versus dietary intervention in obese diabetic subjects despite identical weight loss. Sci Transl Med 3, 80re82 (2011).

26. M. Lips et al., Roux-en-Y gastric bypass surgery, but not calorie restriction, reduces plasma branched-chain amino acids in obese women independent of weight loss or the presence of type 2 diabetes. Diabetes care 37, 3150-3156 (2014).

27. N. Wijayatunga et al., Roux-en-Y gastric bypass surgery alters serum metabolites and fatty acids in patients with morbid obesity. Diabetes/metabolism research and reviews, (2018).

28. T. Arora et al., Roux-en-Y Gastric Bypass Surgery Induces Early Plasma Metabolomic and Lipidomic Alterations in Humans Associated with Diabetes Remission. PLoS One 10, e0126401 (2015).

29. H. Liu, C. Hu, X. Zhang, W. Jia, Role of gut microbiota, bile acids and their cross-talk in the effects of bariatric surgery on obesity and type 2 diabetes. Journal of diabetes investigation 9, 13-20 (2018).

30. M. E. Patti et al., Serum Bile Acids Are Higher in Humans With Prior Gastric Bypass: Potential Contribution to Improved Glucose and Lipid Metabolism. Obesity (Silver.Spring.), (2009).

31. K. K. Ryan et al., FXR is a molecular target for the effects of vertical sleeve gastrectomy. Nature 509, 183-188 (2014).

32. J. Korner et al., Prospective study of gut hormone and metabolic changes after adjustable gastric banding and Roux-en-Y gastric bypass. Int J Obes (Lond) 33, 786-795 (2009).

33. D. Magouliotis, V. Tasiopoulou, E. Sioka, C. Chatedaki, D. Zacharoulis, Impact of Bariatric Surgery on Metabolic and Gut Microbiota Profile: a Systematic Review and Meta-analysis. Obesity surgery 27, 1345-1357 (2017).

34. Z. E. Ilhan et al., Distinctive microbiomes and metabolites linked with weight loss after gastric bypass, but not gastric banding. The ISME journal 11, 2047-2058 (2017).

35. J. Aron-Wisnewsky et al., Major microbiota dysbiosis in severe obesity: fate after bariatric surgery. Gut, (2018).

36. F. Halperin et al., Roux-en-Y gastric bypass surgery or lifestyle with intensive medical management in patients with type 2 diabetes: feasibility and 1-year results of a randomized clinical trial. JAMA Surg 149, 716-726 (2014).

37. T. Jewison et al., SMPDB 2.0: big improvements to the Small Molecule Pathway Database. Nucleic acids research 42, D478-D484 (2014).

38. C. J. Rhodes et al., Plasma proteome analysis in patients with pulmonary arterial hypertension: an observational cohort study. Lancet Respir Med 5, 717-726 (2017).

39. Q. Yang et al., Serum retinol binding protein 4 contributes to insulin resistance in obesity and type 2 diabetes. Nature 436, 356-362 (2005).

40. D. P. MacKinnon, C. M. Lockwood, J. M. Hoffman, S. G. West, V. Sheets, A Comparison of Methods to Test Mediation and Other Intervening Variable Effects. Psychological methods 7, 83 (2002). 
41. R. Barfield et al., Testing for the indirect effect under the null for genome - wide mediation analyses. Genetic Epidemiology 41, 824-833 (2017).

42. Y.-T. Huang, Joint significance tests for mediation effects of socioeconomic adversity on adiposity via epigenetics. Ann Appl Stat 12, 1535-1557 (2018).

43. D. P. Mackinnon, C. M. Lockwood, J. Williams, Confidence Limits for the Indirect Effect: Distribution of the Product and Resampling Methods. Multivariate Behav Res 39, 99 (2004).

44. M. Ritchie et al., limma powers differential expression analyses for RNA-sequencing and microarray studies. Nucleic acids research 43, gkv007-e047 (2015).

45. D. Tingley, T. Yamamoto, K. Hirose, L. Keele, K. Imai, mediation :r package for causal mediation analysis. Journal of statistical software 59, (2014).

46. H. Zhang et al., Estimating and testing high-dimensional mediation effects in epigenetic studies. Bioinformatics 32, 3150-3154 (2016).

47. O. Y. Chén et al., High-dimensional multivariate mediation with application to neuroimaging data. Biostatistics 19, 121-136 (2018).

48. Y. T. Huang, W. C. Pan, Hypothesis test of mediation effect in causal mediation model with high - dimensional continuous mediators. Biometrics 72, 402-413 (2015).

49. V. Djordjilović et al., Global test for high-dimensional mediation: Testing groups of potential mediators. Statistics in Medicine 38, 3346-3360 (2019).

50. M. H. Aguiar-Oliveira, A. Bartke, Growth hormone deficiency: health and longevity. Endocrine Reviews 40, 575-601 (2019).

51. S. Kurtoğlu, N. Hatipoglu, Growth hormone insensitivity: diagnostic and therapeutic approaches. J Endocrinol Invest 39, 19-28 (2016).

52. M. H. Pedersen et al., Substrate Metabolism and Insulin Sensitivity During Fasting in Obese Human Subjects: Impact of GH Blockade. J Clin Endocrinol Metab 102, 13401349 (2017).

53. D. Wu, G. Smyth, Camera: a competitive gene set test accounting for inter-gene correlation. Nucleic acids research 40, e133-e133 (2012).

54. D. A. de Luis et al., Clinical results and nutritional consequences of biliopancreatic diversion: three years of follow-up. Ann Nutr Metab 53, 234-239 (2008).

55. E. T. Aasheim et al., Vitamin status after bariatric surgery: a randomized study of gastric bypass and duodenal switch. Am J Clin Nutr 90, 15-22 (2009).

56. S. Pereira, C. Saboya, G. Chaves, A. Ramalho, Class III obesity and its relationship with the nutritional status of vitamin A in pre- and postoperative gastric bypass. Obes Surg 19, 738-744 (2009).

57. T. E. Graham et al., Retinol-binding protein 4 and insulin resistance in lean, obese, and diabetic subjects. $N$ Engl J Med 354, 2552-2563 (2006).

58. R. V. Shah et al., Proteins altered by surgical weight loss highlight biomarkers of insulin resistance in the community. Arteriosclerosis, Thrombosis, and Vascular Biology 39, 107-115 (2019).

59. B. Janssen et al., Carnosine as a protective factor in diabetic nephropathy: association with a leucine repeat of the carnosinase gene CNDP1. Diabetes 54, 2320-2327 (2005).

60. D. Ben-Zvi et al., Time-Dependent Molecular Responses Differ between Gastric Bypass and Dieting but Are Conserved Across Species. Cell Metab 28, 310-323 e316 (2018). 
61. P. Husek, Z. Svagera, F. Vsiansky, J. Franekova, P. Simek, Prolyl-hydroxyproline dipeptide in non-hydrolyzed morning urine and its value in postmenopausal osteoporosis. Clin Chem Lab Med 46, 1391-1397 (2008).

62. E. W. Yu et al., Effects of Gastric Bypass and Gastric Banding on Bone Remodeling in Obese Patients With Type 2 Diabetes. J Clin Endocrinol Metab 101, 714-722 (2016).

63. M. R. Crawford et al., Increased Bone Turnover in Type 2 Diabetes Patients Randomized to Bariatric Surgery Versus Medical Therapy at 5 Years. Endocr Pract 24, 256-264 (2018).

64. J. A. Minaguchi et al., Remodeling of rat stromal-vascular cells to brite/beige adipocytes by prolyl-hydroxyproline. The Journal of veterinary medical science 79, 547-553 (2017).

65. T. Wang et al., Metabolite profiles and the risk of developing diabetes. Nature Medicine 17, 448-453 (2011).

66. C. B. Newgard et al., A branched-chain amino acid-related metabolic signature that differentiates obese and lean humans and contributes to insulin resistance. Cell Metabolism 9, 311-326 (2009).

67. P. J. White et al., Branched-chain amino acid restriction in Zucker-fatty rats improves muscle insulin sensitivity by enhancing efficiency of fatty acid oxidation and acylglycine export. Molecular metabolism 5, 538-551 (2016).

68. C. Jang et al., A branched-chain amino acid metabolite drives vascular fatty acid transport and causes insulin resistance. Nat Med 22, 421-426 (2016).

69. C. M. Viegas et al., Evidence that 3-hydroxyisobutyric acid inhibits key enzymes of energy metabolism in cerebral cortex of young rats. International journal of developmental neuroscience : the official journal of the International Society for Developmental Neuroscience 26, 293-299 (2008).

70. O. Hamdy et al., Long-term effect of intensive lifestyle intervention on cardiovascular risk factors in patients with diabetes in real-world clinical practice: a 5-year longitudinal study. BMJ Open Diabetes Res Care 5, e000259 (2017).

71. L. Gold, J. Walker, S. Wilcox, S. Williams, Advances in human proteomics at high scale with the SOMAscan proteomics platform. New Biotechnology 29, 543-549 (2012).

72. Q. Yang et al., Serum retinol binding protein 4 contributes to insulin resistance in obesity and type 2 diabetes. Nature. 436, 356-362 (2005).

73. W. E. Gall et al., alpha-hydroxybutyrate is an early biomarker of insulin resistance and glucose intolerance in a nondiabetic population. PLoS One 5, e10883 (2010).

74. R. Gentleman et al., Bioconductor: open software development for computational biology and bioinformatics. Genome Biology 5, 1-16 (2004).

75. D. Wu et al., ROAST: rotation gene set tests for complex microarray experiments. Bioinformatics (Oxford, England) 26, 2176-2182 (2010).

\section{Acknowledgements}

We thank Danyel Cavazos for help with figures; Grace Daher, G. H. Nguyen, Ronaldo Da Silva Francisco Junior, and Lado Gholijashvili for assistance with R package development; Vera 
Djordjilovic for helpful discussion on Hitman; and Harvard Medical School Research Computing for computational resources.

\section{Funding}

This work was supported by unrestricted grant support from Medimmune (to MEP) and the Charles King Trust (fellowship to YY). The parent trial was supported by National Institute of Diabetes and Digestive and Kidney Diseases grants RC1DK086918, R56DK095451, and P30DK03836. JD, HP, SK were also supported by P30DK03836. BK acknowledges support from R01DK43051 and P30DK57521. We also thank the Herbert Graetz Fund at Joslin Diabetes Center and Patient-Centered Outcomes Research Institute (PCORI) grant CE-13046756. Covidien provided funds for the surgical costs of participants with BMI less than 35 who were randomized to undergo surgery; Lifescan, a Division of Johnson \& Johnson, provided home glucose monitoring supplies; Nestle Nutrition Inc. provided Boost; and NovoNordisk provided drug supplies.

\section{Competing Interests}

All authors have completed the ICMJE uniform disclosure form at www.icmje.org/coi_disclosure.pdf and declare: investigator-initiated grant support from the National Institute of Health, National Institute of Diabetes and Digestive and Kidney Diseases (NIDDK), Covidien, and the Herbert Graetz Fund for the submitted work, with supplies from Lifescan, a Division of Johnson and Johnson, Nestle Inc, and Novo Nordisk. YS, BH, JG, and CMR are employees of Medimmune. AK was an employee of Medimmune when the work was initiated, but is now employed at Sanofi. ABG reports this work was initiated when employed at the Joslin Diabetes Center but is now an employee of Novartis Institutes of Biomedical Research. She has also received research grants and honorariums from the National Heart, Lung, and Blood Institute and the American Diabetes Association, and served on advisory boards for the NIDDK, Baranova, and Kowa. DCS received funds from PCORI. DCS is a stock/shareholder of GI Windows, and is a scientific advisory board member for GI Windows and Medtronic. MEP received unrestricted investigator-initiated research grant funding from Medimmune to support the present work, and assay funding from SomaLogic. No other relationships or activities occurred that could appear to have influenced the submitted work.

\section{Trial Registration}

Clinicaltrials.gov NCT01073020 
Figure 1

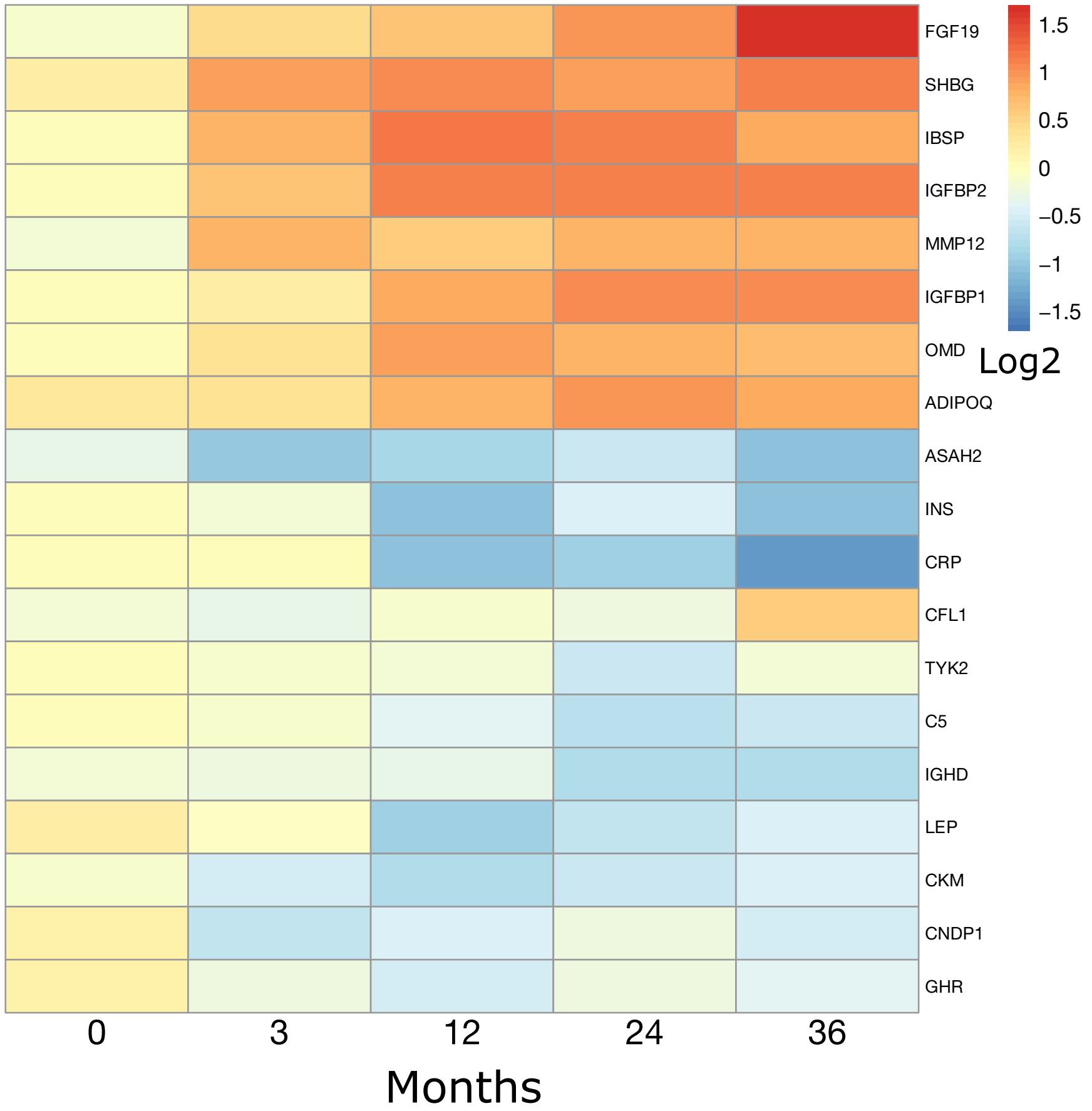


Figure 2

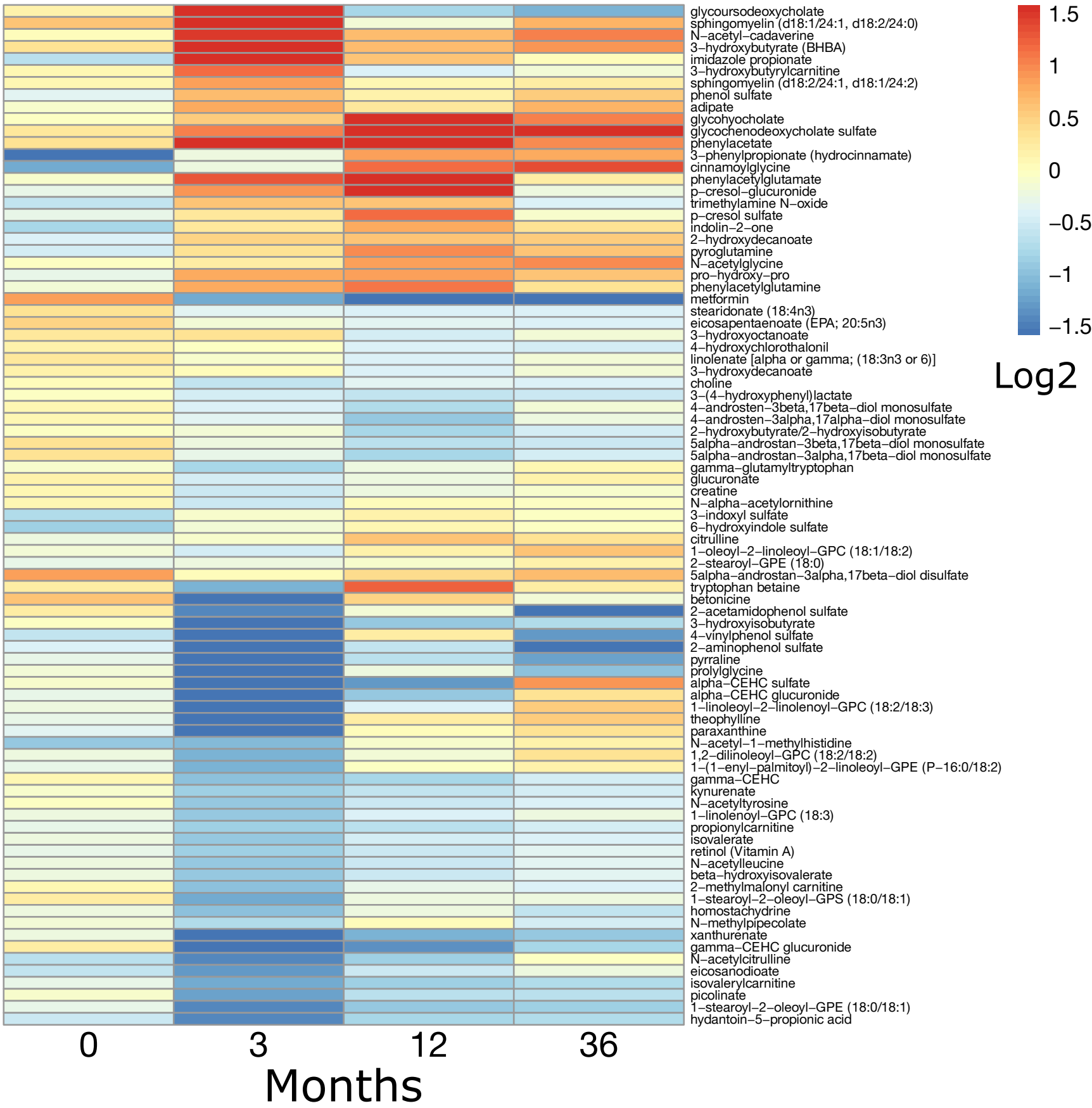




\section{Figure 3.}

A.

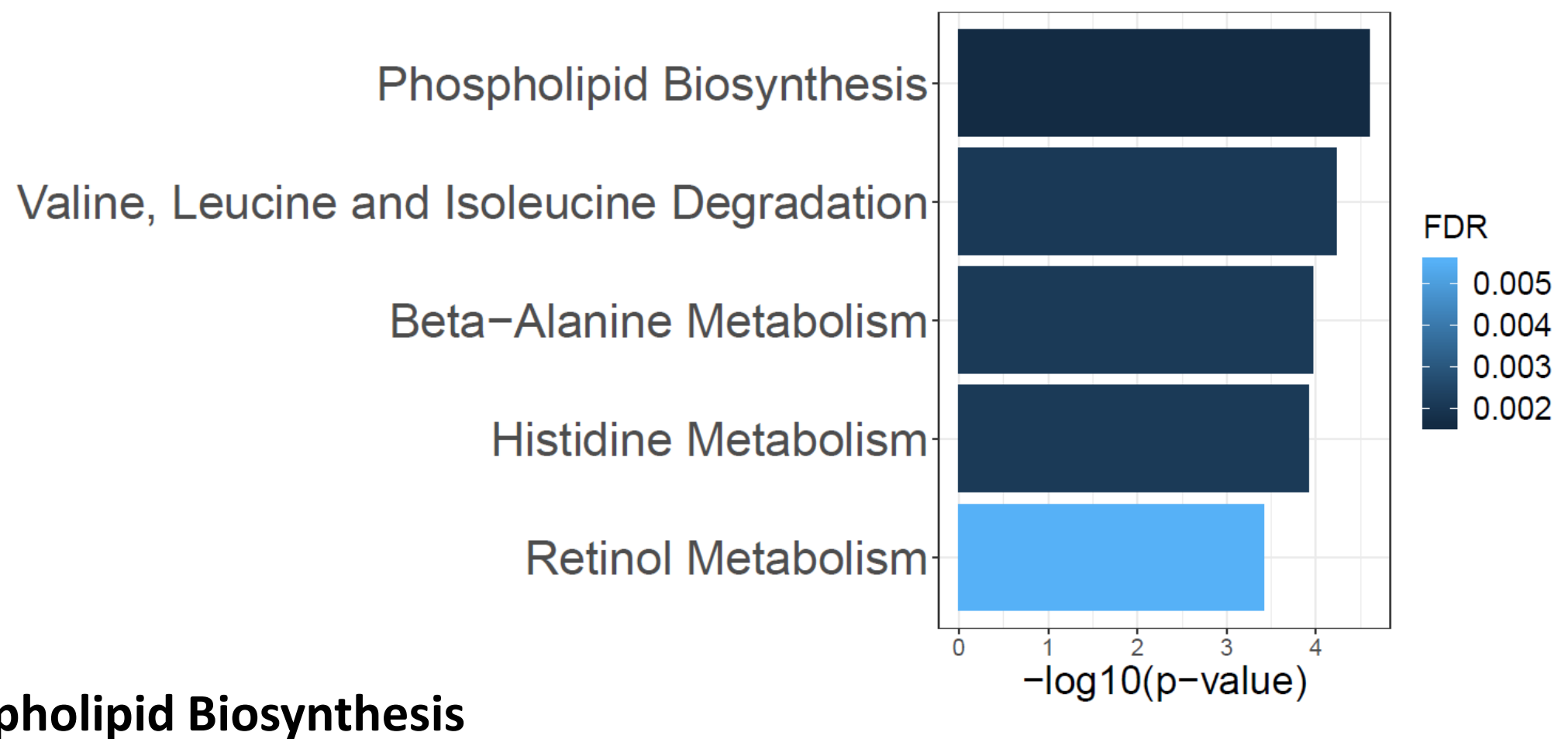

B. Phospholipid Biosynthesis

choline phosphate

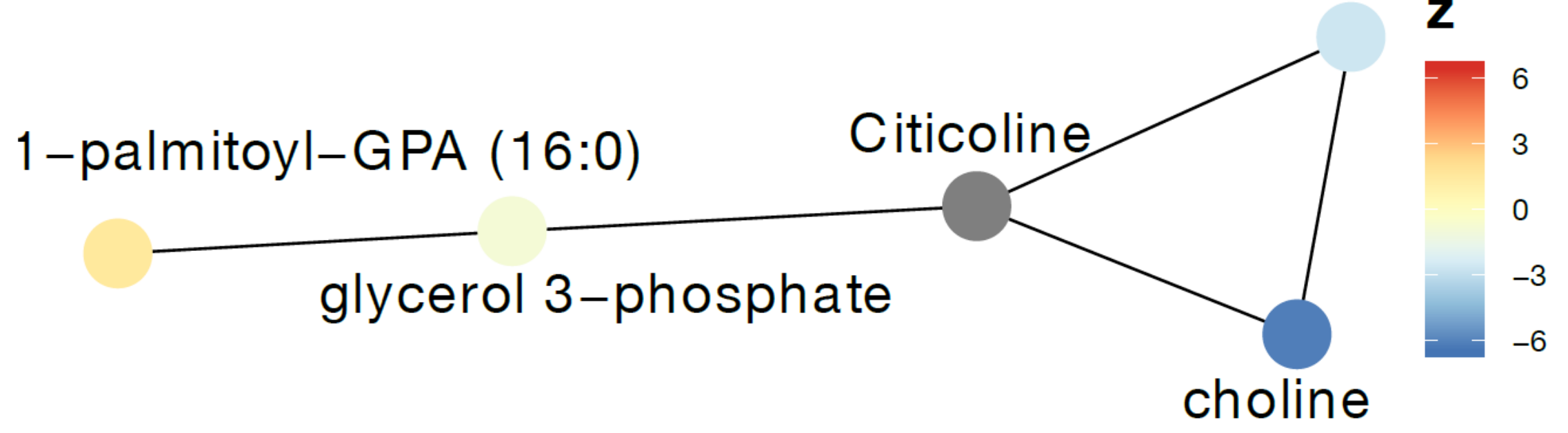


Figure 5.

A. Beta-alanine metabolism

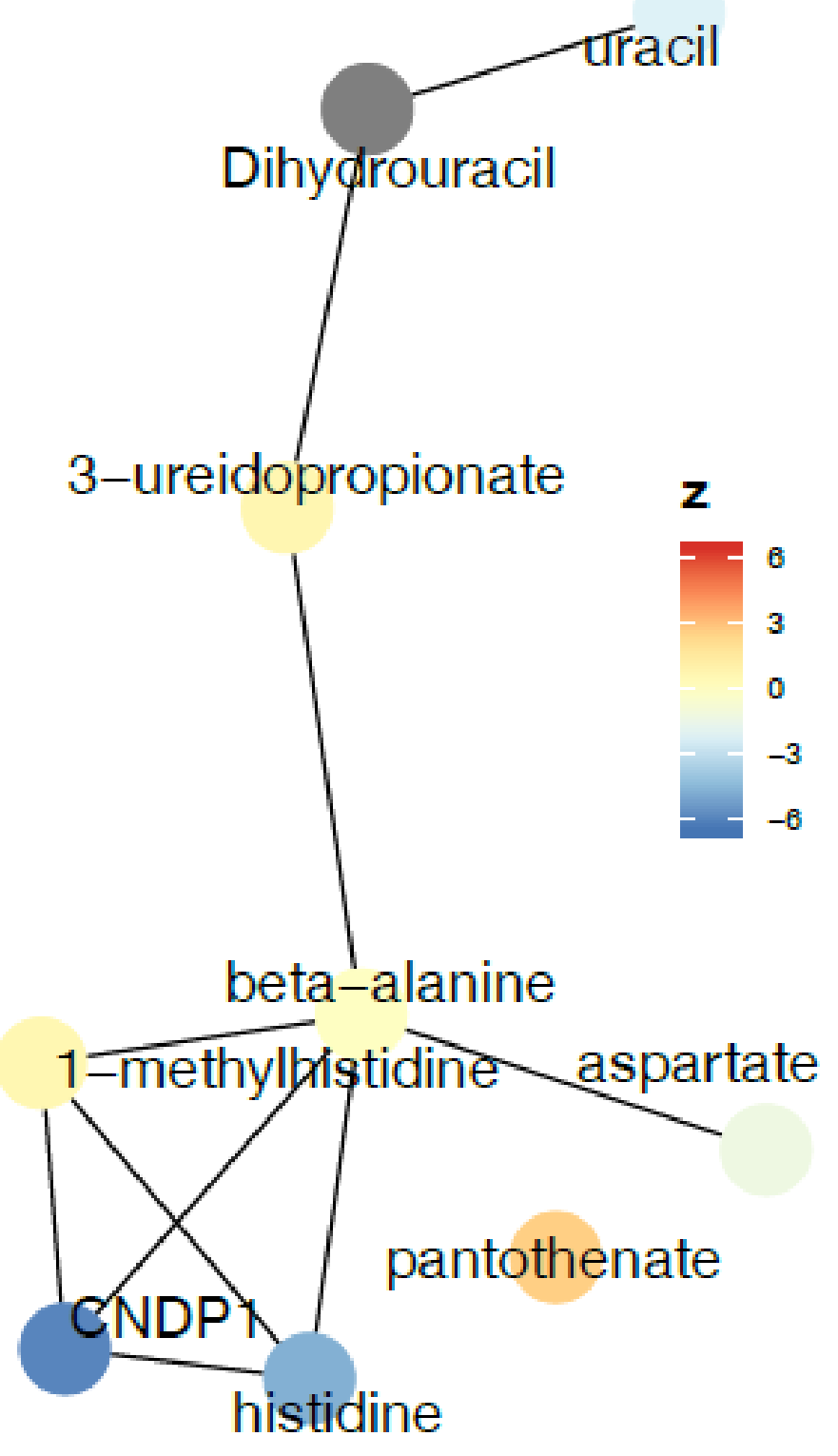

B.

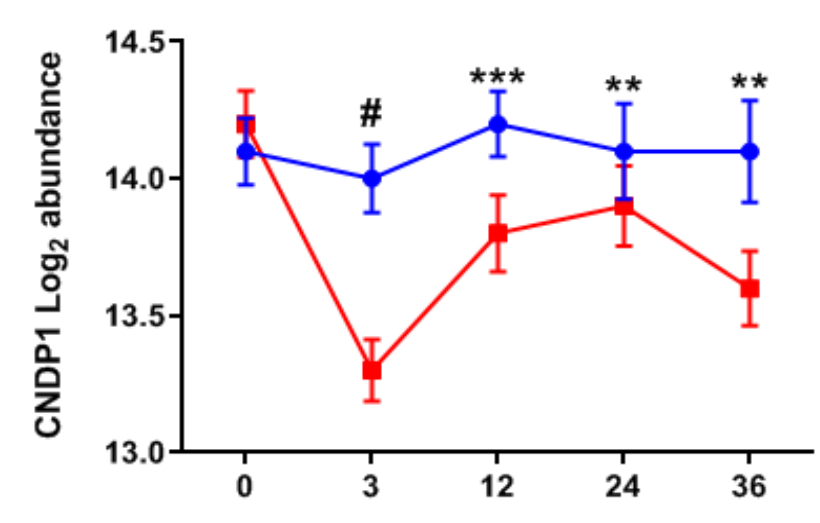

$\rightarrow$ RYGB

$\rightarrow$ DWM

c.
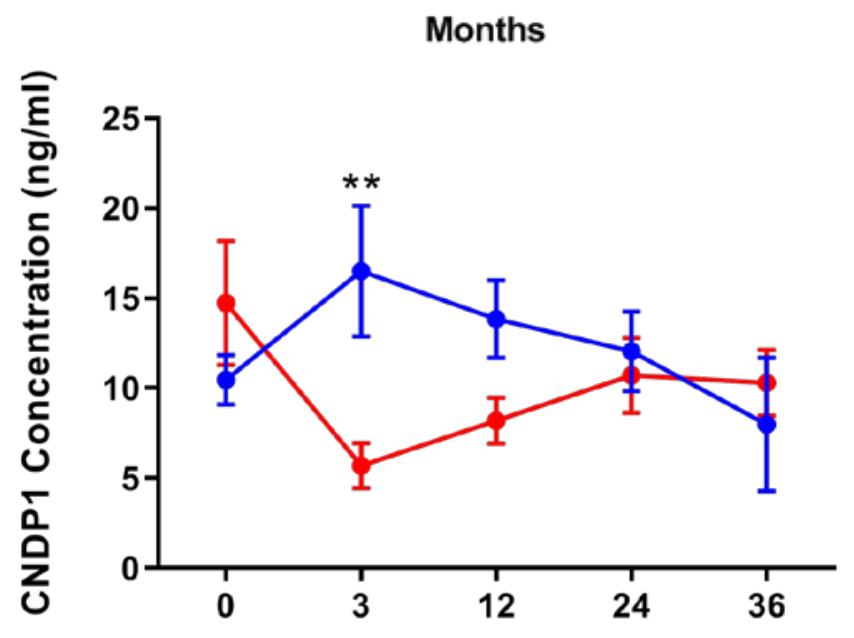

D.

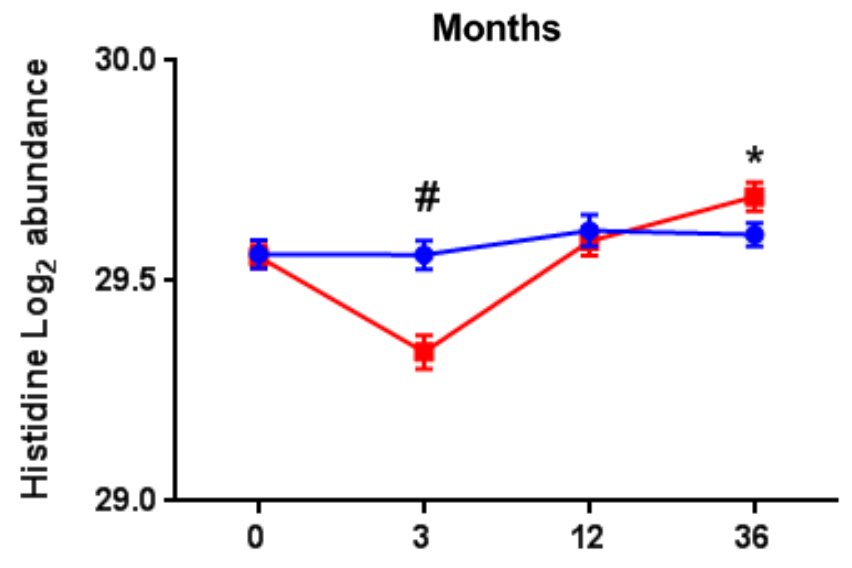


Figure 6.

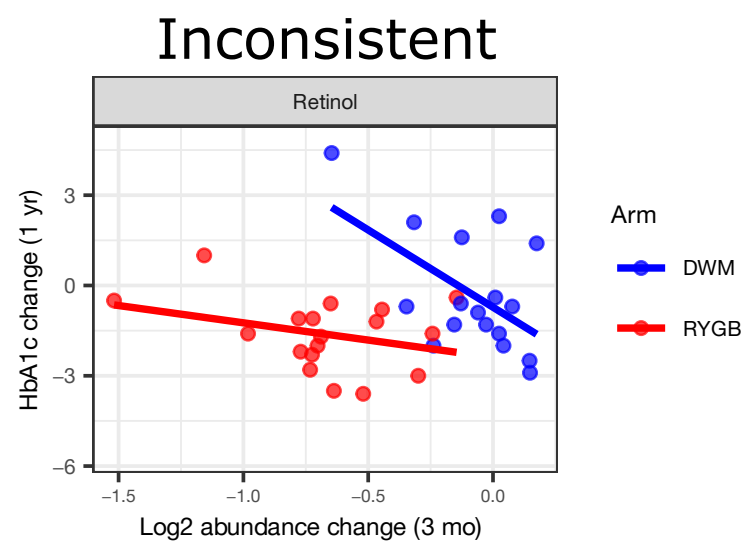

Consistent mediators

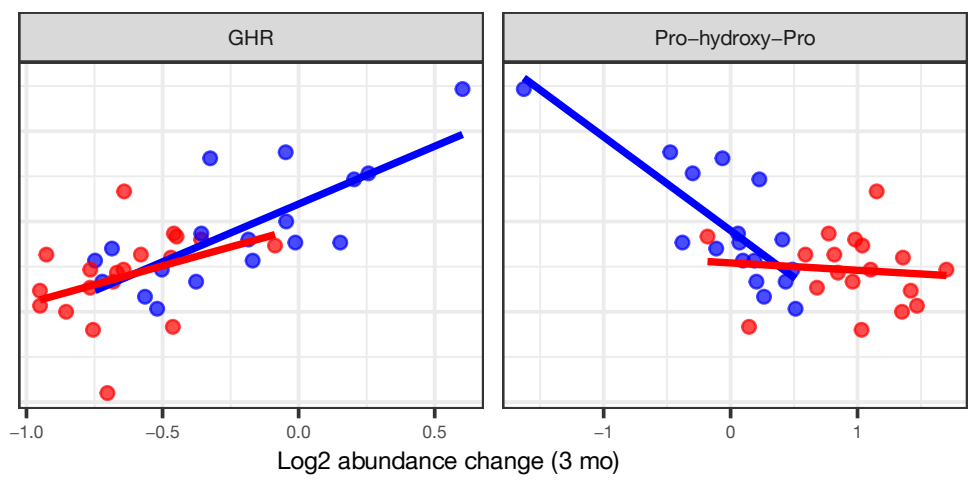




\section{Figure S1.}

A.

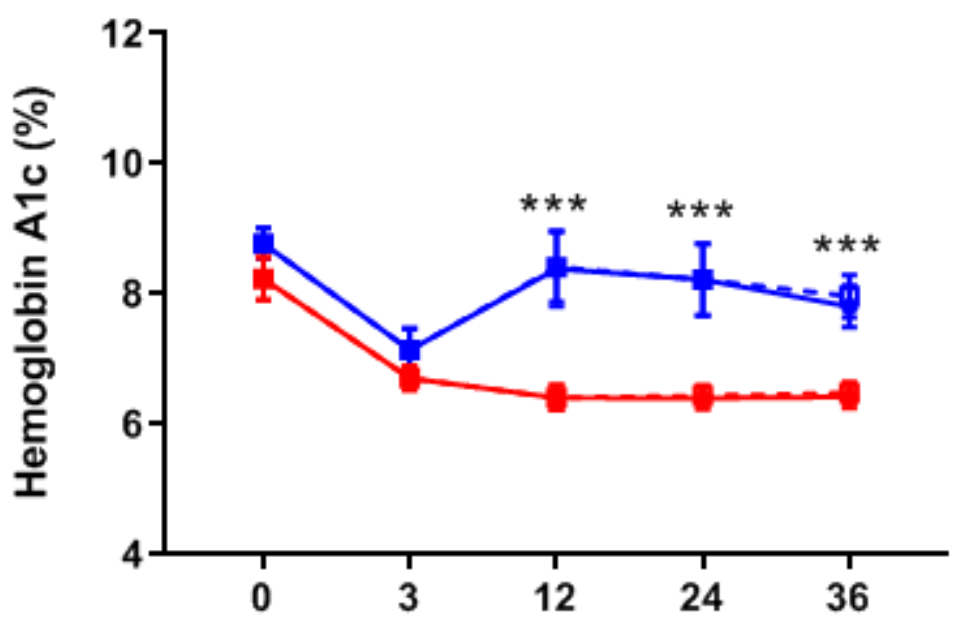

$\rightarrow$ RYGB Omics

-в · RYGB Total

$\rightarrow$ DWM Omics

-в. DWM Total

Months

B.

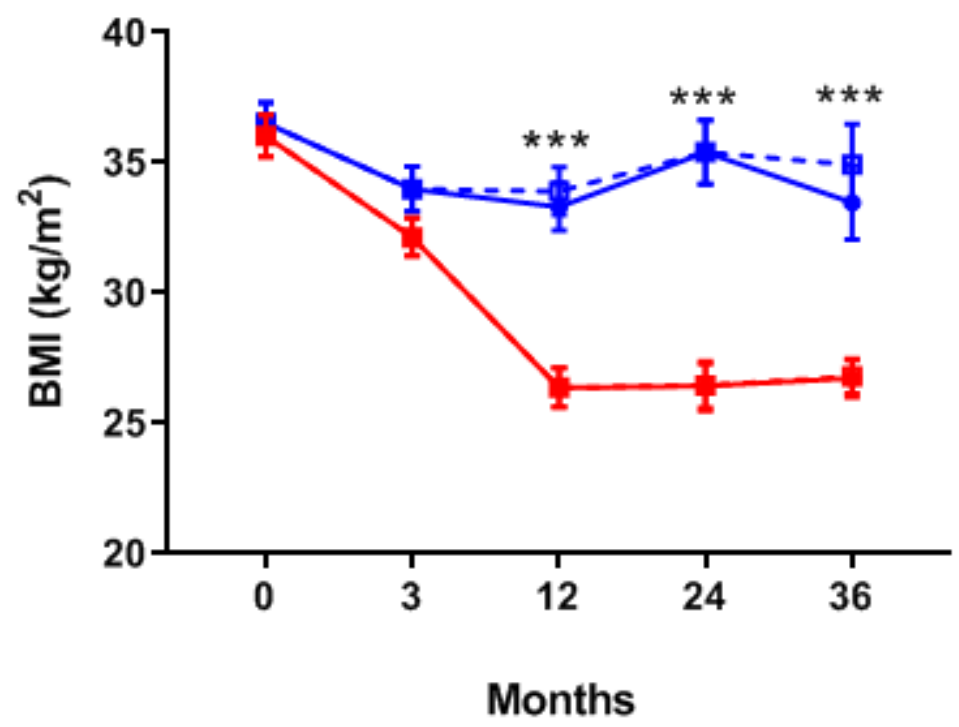

$\rightarrow$ RYGB Omics

-в. RYGB Total

$\rightarrow$ DWM Omics

-в. DWM Total

Months 
Figure S2.

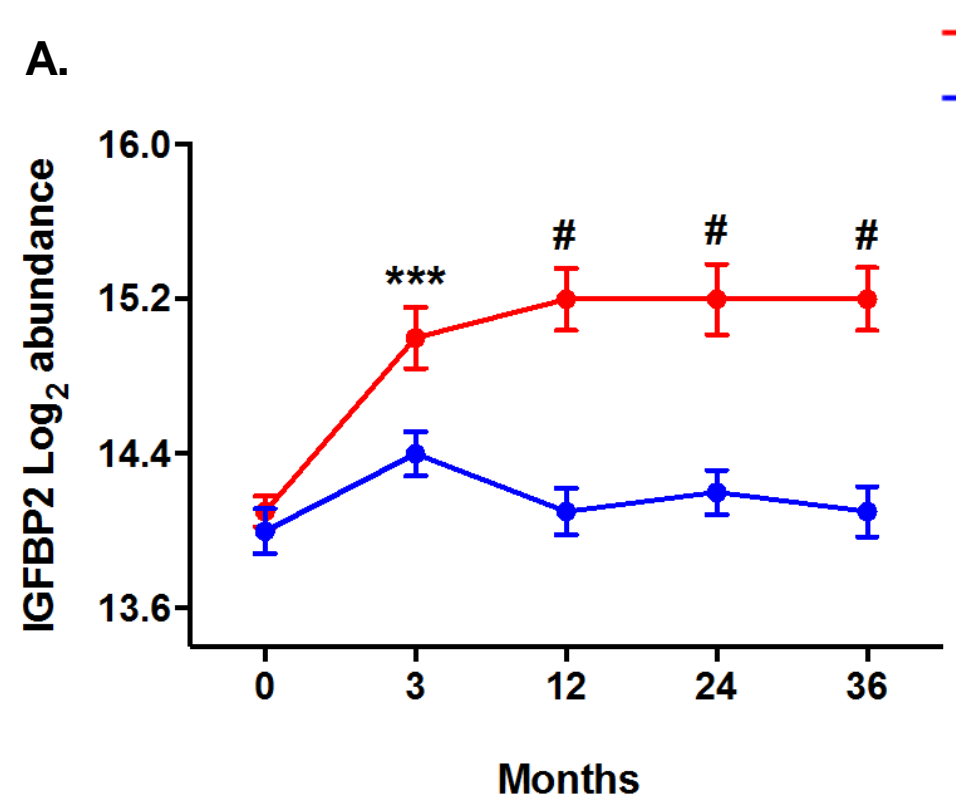

c.
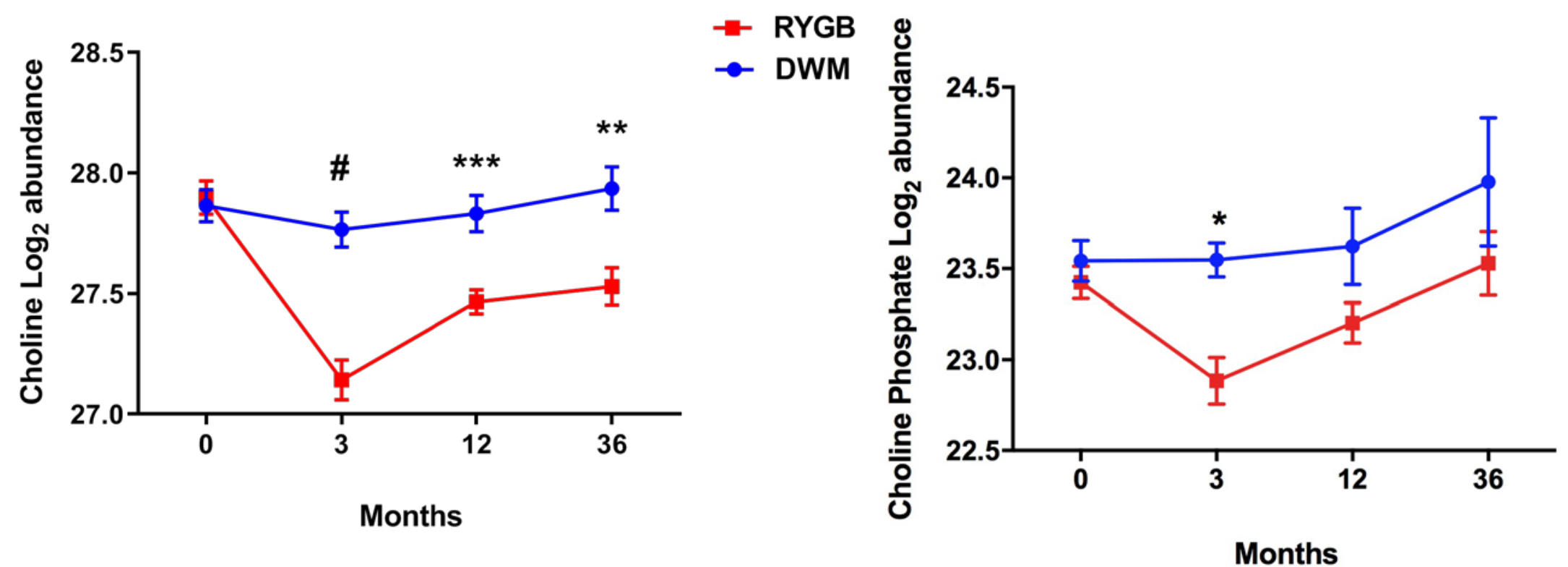

\section{Months}


Figure S3.

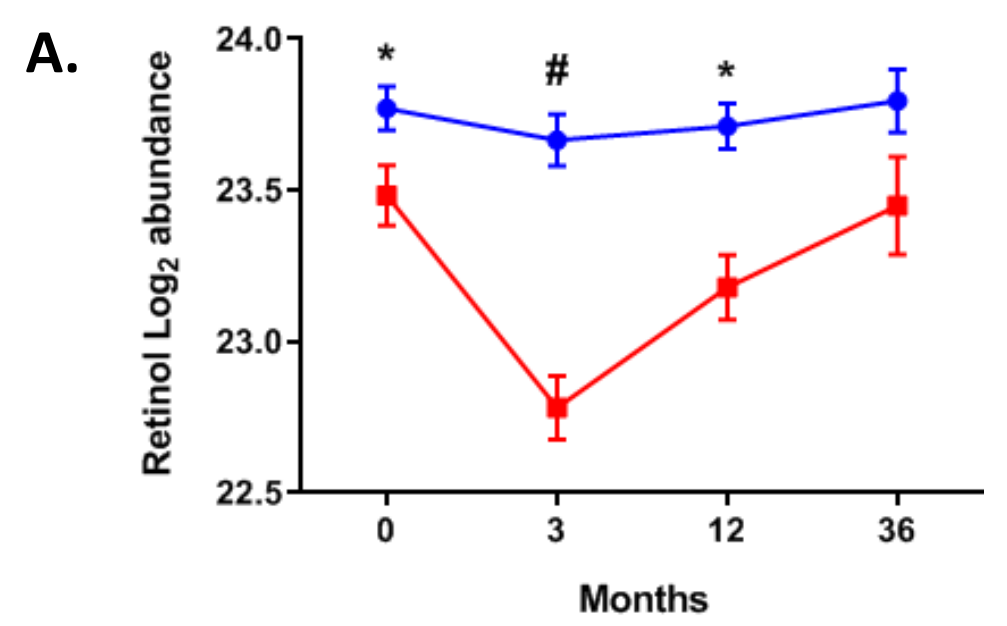

-

$\rightarrow$ DWM B.
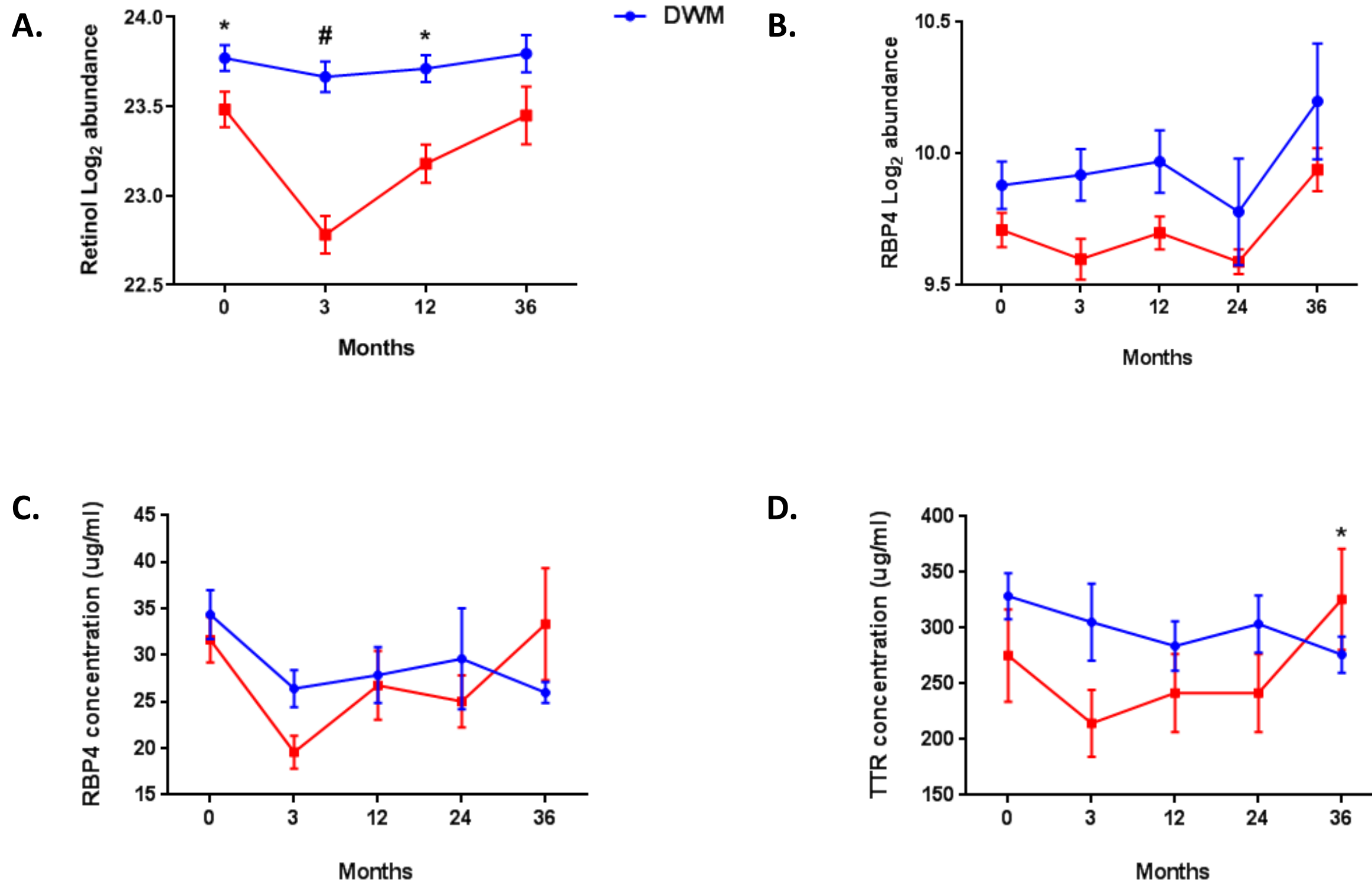

D.

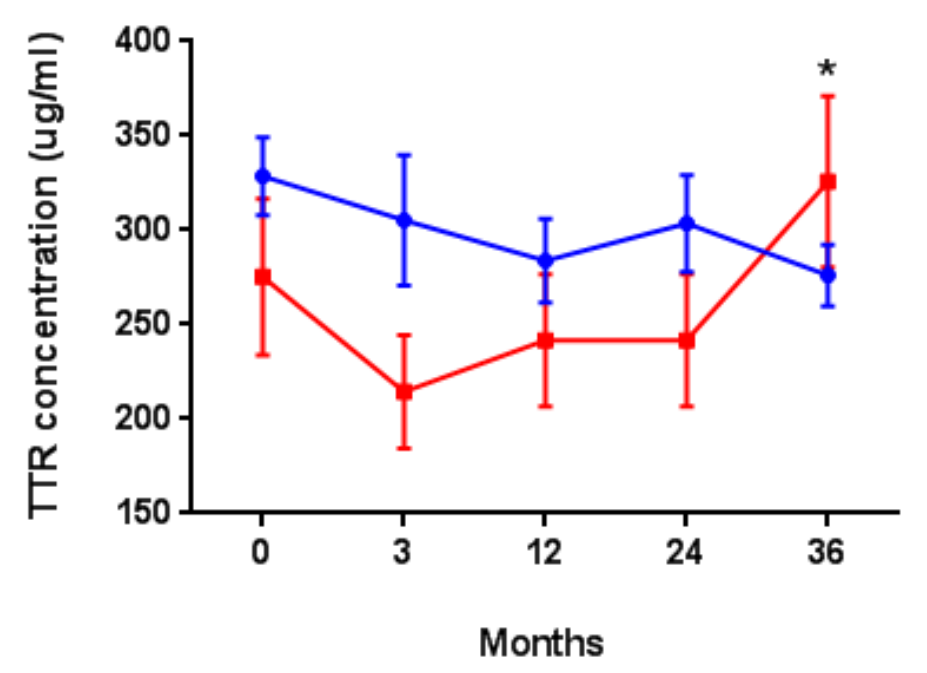


Figure S4.

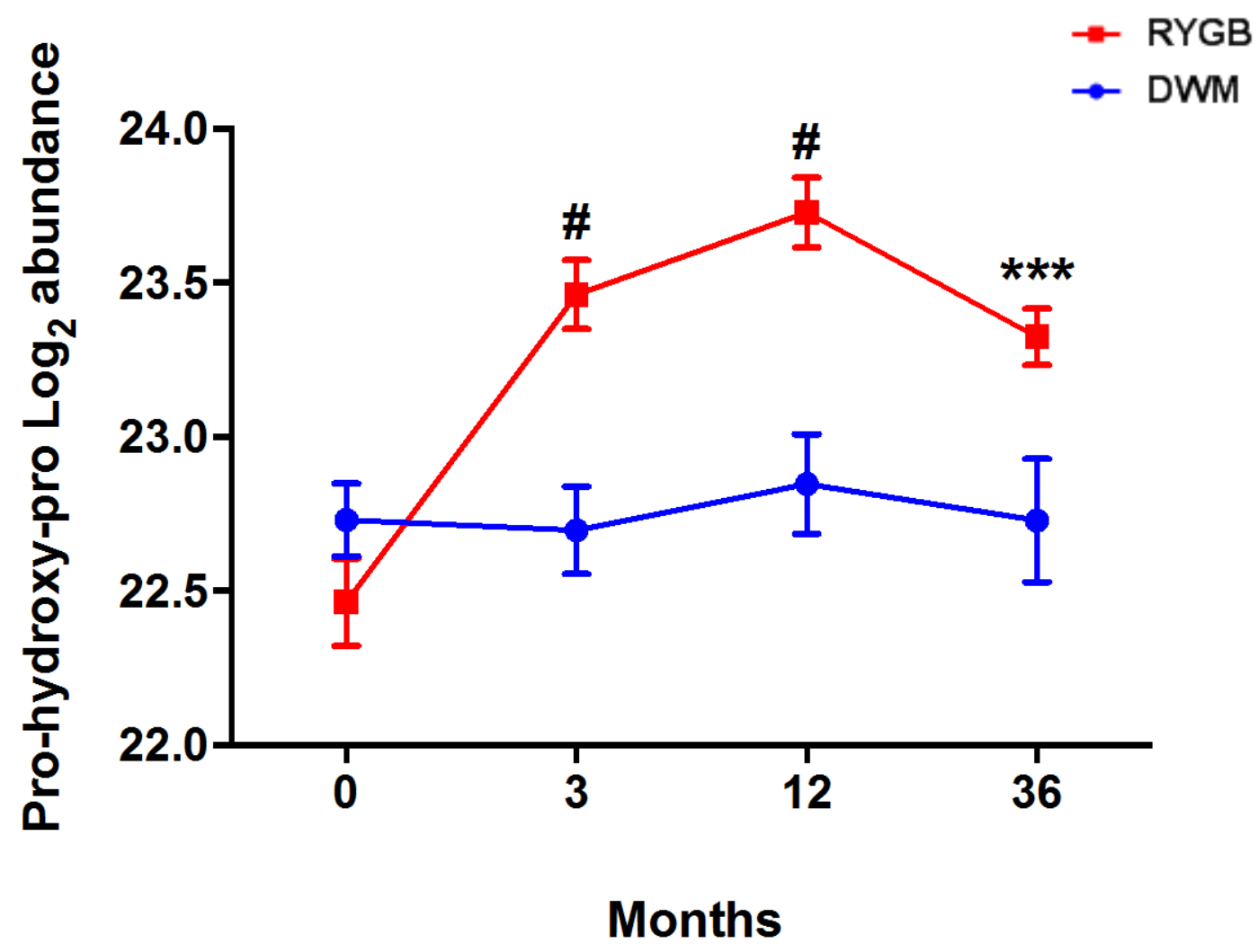




\section{Figure S5.}

A.

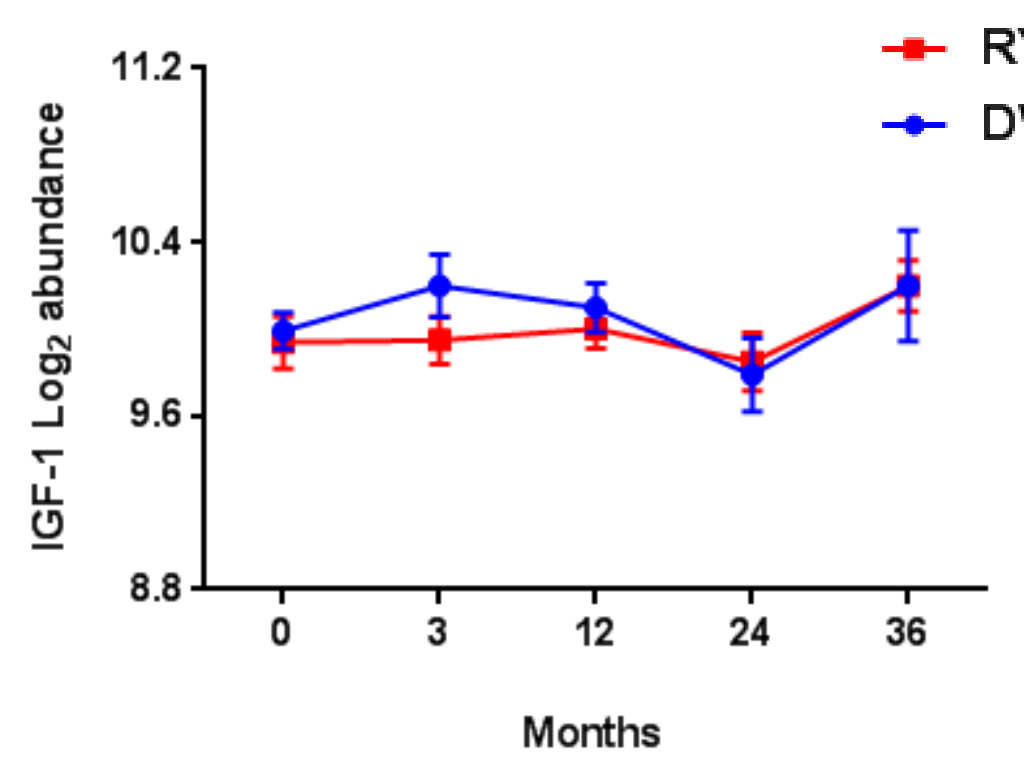

C.

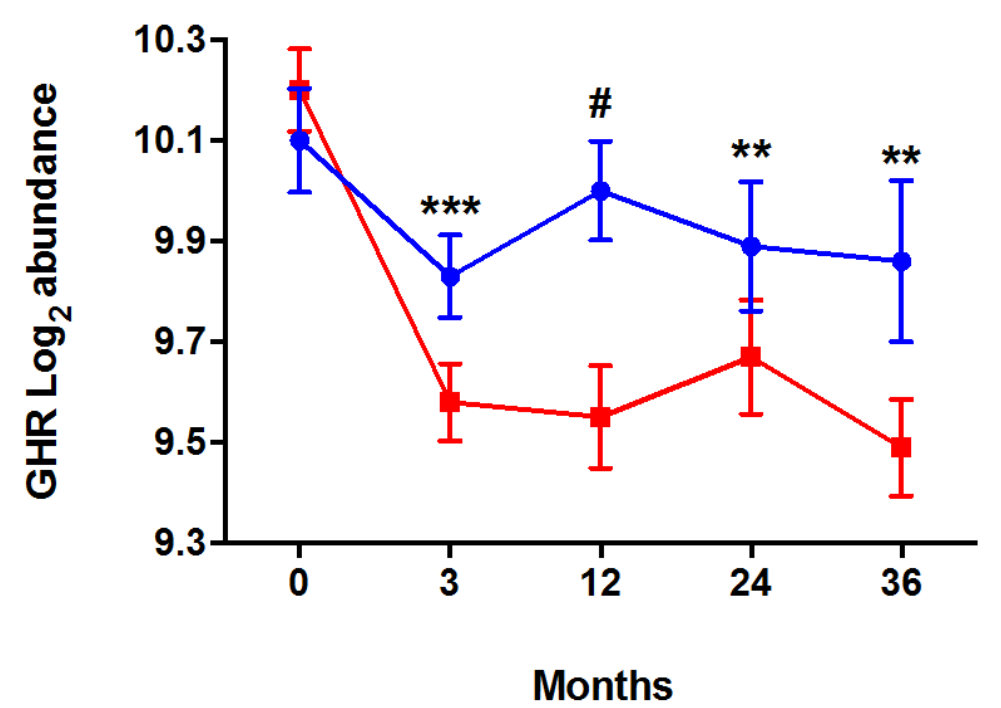

B.

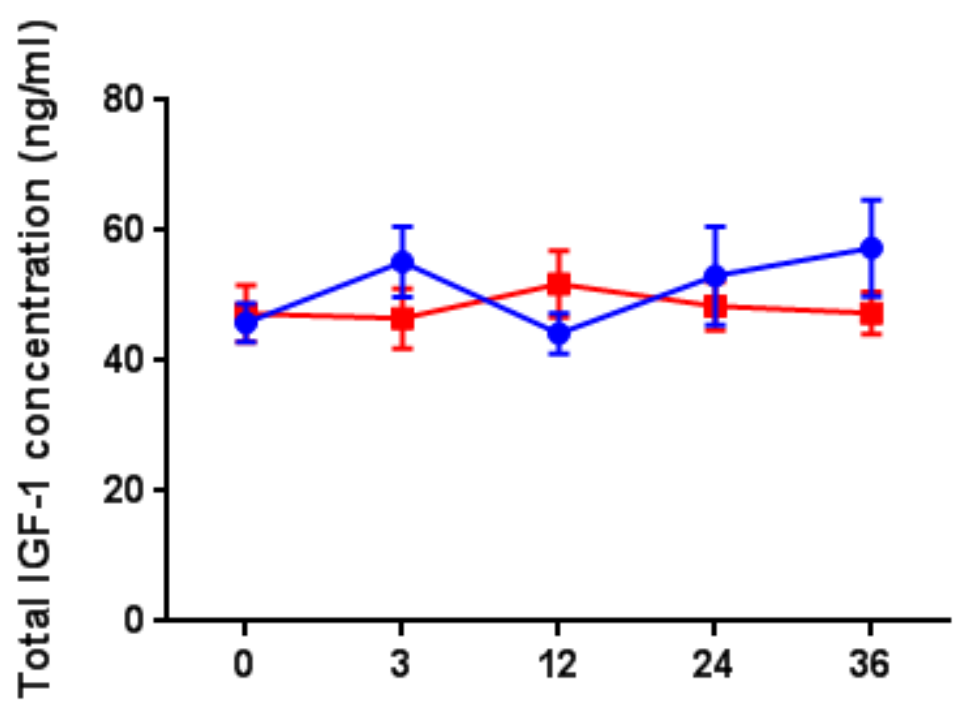

D.

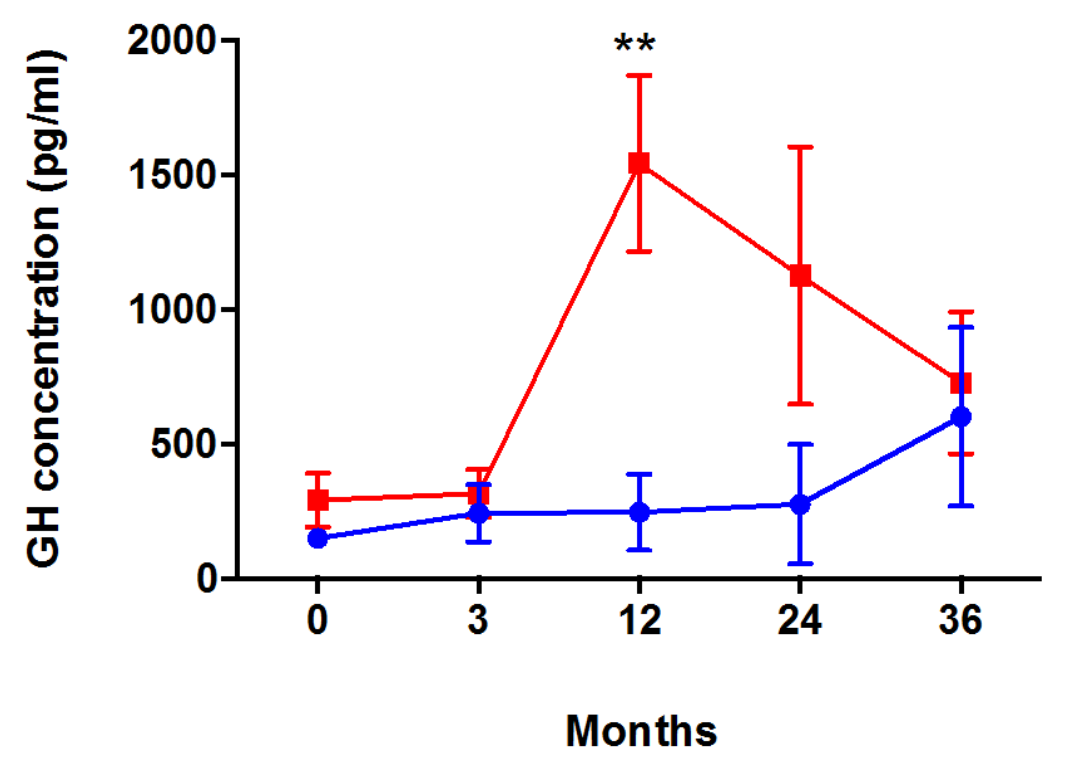


Figure S6.

Roux en $Y$

Gastric Bypass

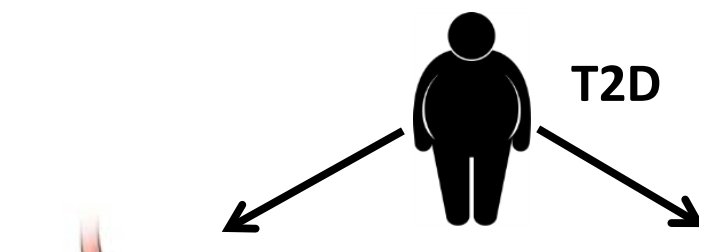

Randomization

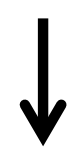

,

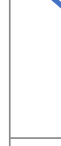

$\begin{array}{llll}0 & 3 & 12 & 36\end{array}$

Months

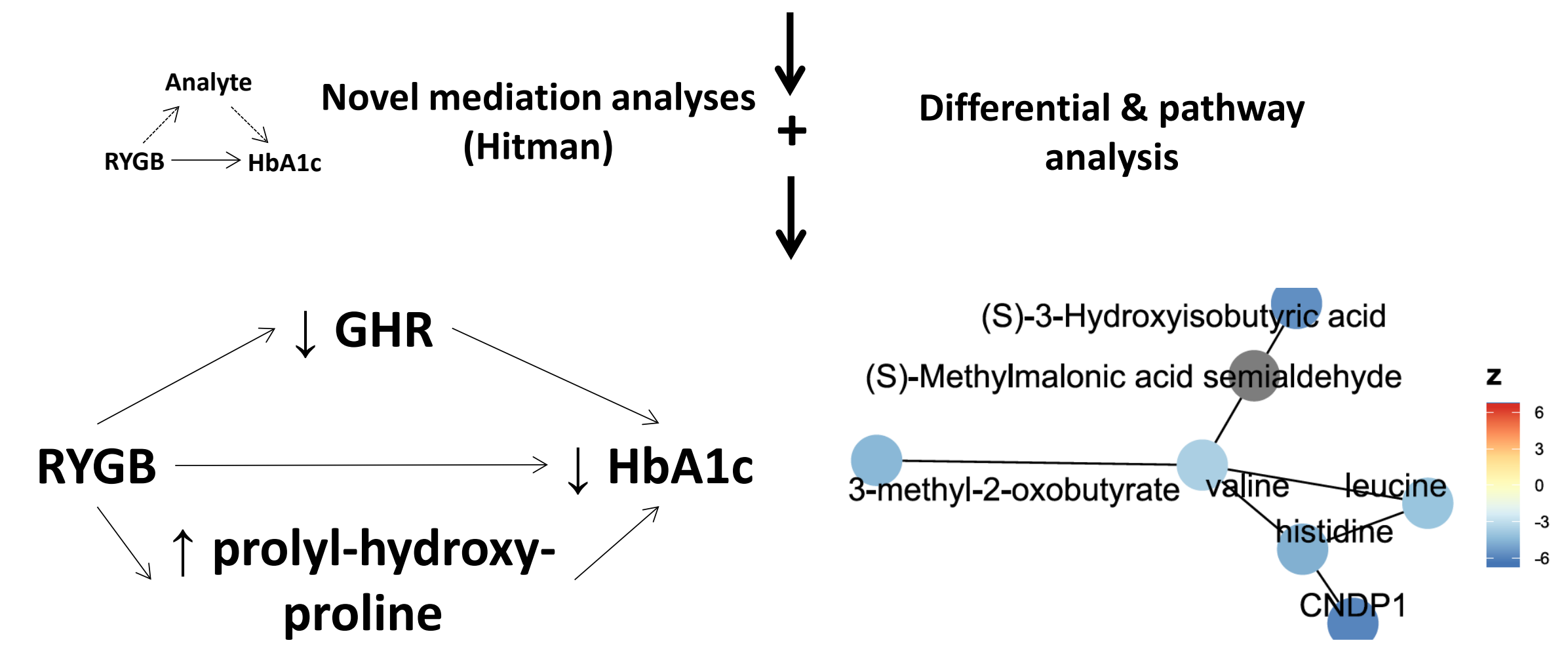

然自

Longitudinal clinical data, plasma proteomics \& metabolomics

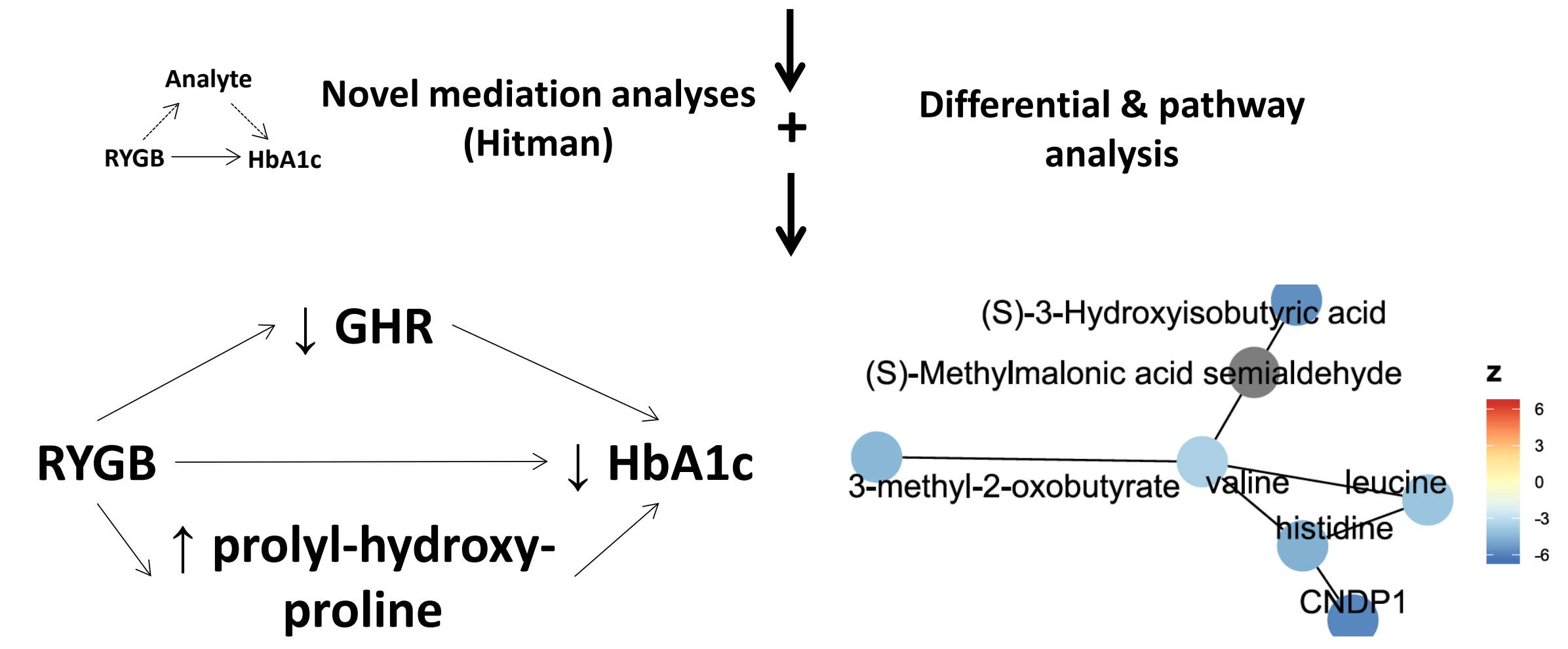

Differential \& pathway analysis
Diabetes and weight management 


\section{Text S1. High-throughput mediation analysis (Hitman)}

\section{Model}

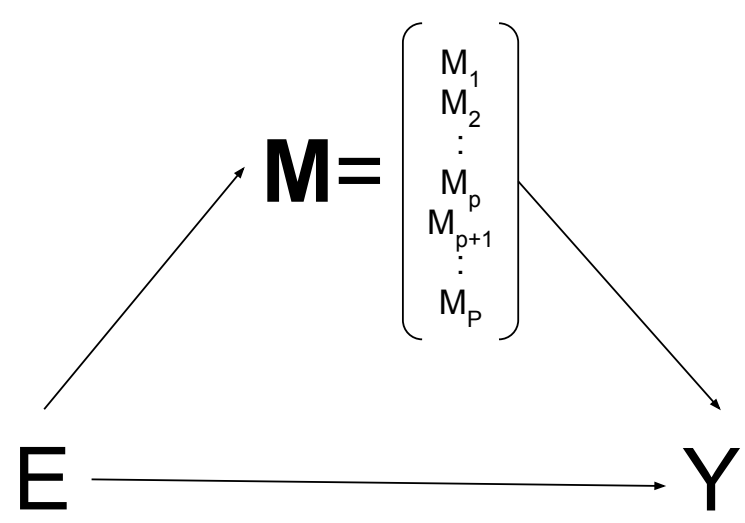

(a)

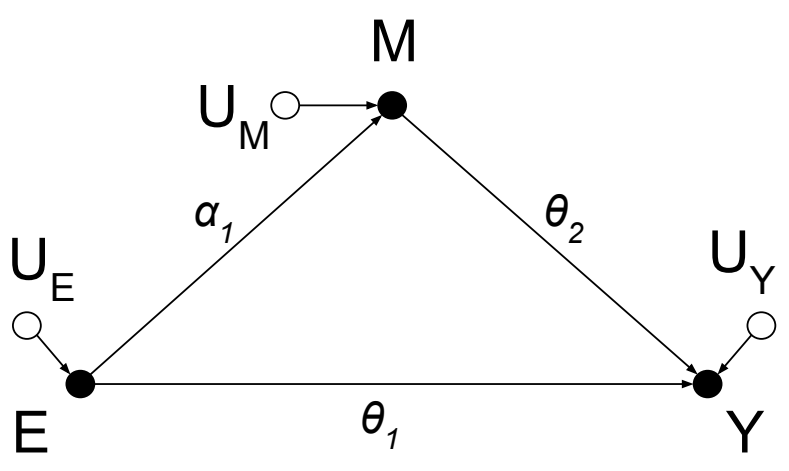

(b)

Figure 1: Hitman model without covariates.

Our causal model of the effect of the treatment or exposure $(E)$ on the outcome $(Y)$, and the effect's mediators $(M)$ are shown in Figure 1a and 1b. We have $E \rightarrow \mathbf{M} \rightarrow Y$ and $E \rightarrow Y$, where arrows $(\rightarrow)$ represent potential directed causal effects, with structural parameters next to the arrows. Our unmediated model is $E \stackrel{\lambda_{1}}{\longrightarrow} Y$, where the structural parameter $\lambda_{1}$ represents the total effect (http://davidakenny.net $/ \mathrm{cm} /$ mediate.htm). Our causal mediation model per mediator is shown in Figure $1 \mathrm{~b}$. We assume that $E$ causally affects $Y$, and that this total effect is robust. We are then interested in the mediation effect (or indirect effect), represented by $\alpha_{1} \theta_{2} . \theta_{1}$ is the direct effect per mediator. Lack of an arrow indicates no causal effect (Pearl 2010), so the model assumes that neither $M$ nor $Y$ affect $E$ (as occurs when $E$ is randomized), and that $Y$ does not affect $M$. Hitman does not test these assumptions.

Our model in Figure 1a is based on a biological system, such as a cell, where an intervention (or exposure)

like a gene knockout can have effects across all activities in the cell. The number of potential mediators of the exposure is $P$, where $P$ is astronomically large. In an omics experiment, we measure $p$ (with $p$ much smaller than $P, p<<P$ ) analytes across $n$ samples, with $p>>n$. The $P$ mediators in Figure 1a have unknown relationships among themselves and on the outcome. Ideally, we would identify these relationships so that we can account for them, but with few samples and an astronomical number of mostly unmeasured mediators, we do not attempt that here, and instead pursue an exploratory, one-at-a-time analysis of our measured mediators, so that we can identify which mediators to pursue with further studies.

Our linear structural equation model (SEM) per mediator with covariate vector or matrix $X$ is:

$$
\begin{aligned}
E & =\nu_{0}+\nu_{1} X+U_{E} \\
M & =\alpha_{0}+\alpha_{1} E+\alpha_{2} X+U_{M} \\
Y & =\theta_{0}+\theta_{1} E+\theta_{2} M+\theta_{3} X+U_{Y}
\end{aligned}
$$

where $U$ terms represent omitted factors that explain sources of variation. The SEM's coefficients represent structural parameters estimable from the data. These terms must be mutually independent for the validity of mediation analyses. When we randomize $E$, we know that $U_{E}$ is independent of the other $U$ 's. However, 
$U_{M}$ is often dependent with $U_{E}$, due to another mediator (likely unmeasured) affecting both $M$ and $Y$. So, "theoretical knowledge must be invoked to identify the sources of these correlations and control for common causes (so called 'confounders') of $M$ and $Y$ whenever they are observable" (Pearl 2014a). Such confounders can be included in $X$. When $X$ is a matrix, $\nu_{1}, \alpha_{2}$, and $\theta_{3}$ are vectors.

We implement Hitman for our data with the assumptions that $E$ is randomized and, under the null, the $U$ 's are independent given $X$ and $U_{M}$ and $U_{Y}$ have known distributions. For example, our analyte mediators and outcome follow the normal distribution. Hitman also accommodates outcomes that follow any generalized linear model, such as logistic regression for binary $Y$.

Hitman is based on the joint significance or causal steps test. The joint significance test requires that the exposure causes the outcome (Judd \& Kenny 1981)(MacKinnon et al. 2002), and has been shown to have more power than the product method and to control its false positive rate, because it is an intersection-union test (Huang et al. 2018). A drawback of the joint significance test is that it doesn't account for direction of effect. For example, if $E$ increases $Y$ (i.e. an increase in $E$ increases $Y$ ), then we would want mediators $M$ where $E$ increases $M$ and $M$ increases $Y$, or where $E$ decreases $M$ and $M$ decreases $Y$. However, the joint significance test might find as significant an "inconsistent" mediator where $E$ increases $M$ and $M$ decreases $Y$ (MacKinnon et al. 2002). We address this in Hitman.

\section{Method}

For each mediator $(M)$, the null hypothesis is that $E$ has no effect on $M\left(\alpha_{1}=0\right)$; or that $M$ has no effect on $Y\left(\theta_{2}=0\right)$; or that the direction of mediation, $\operatorname{sgn}\left(\alpha_{1} \theta_{2}\right)$, is not consistent with the direction of the total effect, $\operatorname{sgn}\left(\lambda_{1}\right)$, with $\operatorname{sgn}$ being the sign function. An example of an inconsistent mediator is $E$ increases $Y$, but $E$ increases $M$ and $M$ decreases $Y$. The alternative hypothesis is that $E$ affects $M$ and $M$ affects $Y$, and that the direction of $E \rightarrow M$ and $M \rightarrow Y$ are consistent with that of the total effect of $E$ on $Y$. This tests if $M$ explains at least some of the dependence between $E$ and $Y ; M$ need not explain all of it.

Hitman implements tests with high-throughput mediators using the R/Bioconductor linear modeling package limma (Ritchie et al. 2015). Limma models the variance of features (e.g. proteins or metabolites) with an empirical Bayesian method, which exploits information about shared technical variance between features for improved power, especially when the sample size is small (Ritchie et al. 2015). Limma can also account for a mean-variance relationship, as occurs in RNA-seq (Law et al. 2014). To model variance of feature abundance in linear modeling, limma models feature abundance as the dependent variable.

To estimate the direction of mediation, Hitman uses $\operatorname{sgn}\left(\hat{\lambda}_{1}\right)$ from the linear model with covariates $Y=$ $\lambda_{0}+\lambda_{1} E+\lambda_{2} X+\epsilon$. This model does not include mediators, because here we are not interested in the indirect or mediated effects. Hitman assumes the total effect is robust, so treats the estimated direction as correct, i.e. $\operatorname{sgn}\left(\hat{\lambda}_{1}\right)=\operatorname{sgn}\left(\lambda_{1}\right)$.

For each mediator $M$ :

1. Measure effect of $E$ and $M$ using equation 2. Here and later, if $X$ is absent, remove its term. If $X$ has multiple columns, $\alpha_{2}$ is a vector. Test $\alpha_{1}=0$ in limma and define the resulting p-value $p_{1}$.

2. Measure association of $M$ and $Y$ given $E$ (and possibly $X$ ) using equation 3. We would like to test $\theta_{2}=0$ using limma. To model the variance of $M$ with empirical Bayesian methods, we need to make $M$ the dependent variable. So we use an approach similar to partial correlation.

i. Estimate the residuals of $Y=\lambda_{0}+\lambda_{1} E+\lambda_{2} X+e$ as $e_{Y}$.

ii. Estimate the residuals of $M=\alpha_{0}+\alpha_{1} E+\alpha_{2} X+e$ as $e_{M}$.

iii. From the linear model $e_{M}=\theta_{2} e_{Y}+\epsilon$, test $\theta_{2}=0$ in limma and define the resulting p-value $p_{2}$. 
Now define

$$
S= \begin{cases}0, & \text { if } \operatorname{sgn}\left(\alpha_{1} \theta_{2}\right) \neq \operatorname{sgn}\left(\lambda_{1}\right) \\ 1, & \text { if } \operatorname{sgn}\left(\alpha_{1} \theta_{2}\right)=\operatorname{sgn}\left(\lambda_{1}\right)\end{cases}
$$

and

$$
\hat{S}= \begin{cases}0, & \text { if } \operatorname{sgn}\left(\hat{\alpha}_{1} \hat{\theta}_{2}\right) \neq \operatorname{sgn}\left(\hat{\lambda}_{1}\right) \\ 1, & \text { if } \operatorname{sgn}\left(\hat{\alpha}_{1} \hat{\theta}_{2}\right)=\operatorname{sgn}\left(\hat{\lambda}_{1}\right) .\end{cases}
$$

3. If $\hat{S}=1$, then the direction of total effect agrees with that of $E \rightarrow M \rightarrow Y$, and the final p-value $=$ $0.5 \max \left(p_{1}, p_{2}\right)$. Otherwise, the direction of the indirect effect is inconsistent with that of the overall effect, and the final p-value $=1-0.5 \max \left(p_{1}, p_{2}\right)$. The intuition behind the test is that $\hat{S}$ allows for one-sided testing of $\hat{\theta}_{2}$ or $\hat{\alpha}_{1}$.

4. To account for testing multiple mediators, calculate false discovery rates (FDRs) from the mediator p-values (Benjamini \& Hochberg 1995).

\section{Example}

We illustrate Hitman through an example of an inconsistent mediator (Figure 2). This figure is reminiscent of Simpson's Paradox (Pearl 2014b). The exposure increases the outcome, and it increases the mediator. Moreover, the mediator is associated with the outcome, but it decreases the outcome, resulting in inconsistent mediation.

To define this generic example, let the realized value of the random estimate $\widehat{\alpha}_{1}$ be $a$, and similarly the realized value of $\widehat{\beta}_{1}$ be $b$, and of $\widehat{\lambda}_{1}$ be $g$. In this example, $a>0, g>0$, and $b<0$. Then $\operatorname{sign}(g) \neq \operatorname{sign}(a b)$. However, this evidence is opposite the alternative hypothesis, where we want the direction of effect to be consistent: $\operatorname{sgn}\left(\lambda_{1}\right)=\operatorname{sign}\left(\alpha_{1} \beta_{1}\right)$. Thus, Hitman conservatively estimates this mediator's p-value as 1 , whereas if consistency was not accounted for, the p-value would be $<0.001$.

\section{Mathematical justification}

We show here that Hitman properly controls its false positive rate in theory. Below we show this in simulations. Hitman, like the joint significance method, requires that the exposure causes the outcome. Hitman also requires that this effect is robust, so the sign of the estimated total effect matches the sign of the total effect.

Then for a single mediator, the null and alternative hypotheses as described above are:

$$
\begin{aligned}
& H_{0}: \alpha_{1}=0 \cup \theta_{2}=0 \cup S=0 \\
& H_{a}: \alpha_{1} \neq 0 \cap \theta_{2} \neq 0 \cap S=1 .
\end{aligned}
$$

But the terms in $H_{a}$ are not independent, because, e.g. $S=1 \rightarrow \alpha_{1} \neq 0 \cap \theta_{2} \neq 0$, and consequently we could also write $H_{a}: S=1$.

To show that Hitman controls its size given the composite null, we consider the most challenging case under $H_{0}$. Without loss of generality, under the assumption that the estimated direction is correct, let $\operatorname{sgn}\left(\hat{\lambda}_{1}\right)=\operatorname{sgn}\left(\lambda_{1}\right)=1$. Hitman treats $\alpha_{1}$ and $\theta_{2}$ symmetrically, so without loss of generality we select $\theta_{2}$ to have a defined sign, and without loss of generality consider $\operatorname{sgn}\left(\theta_{2}\right)=1$. For the case to be maximally 


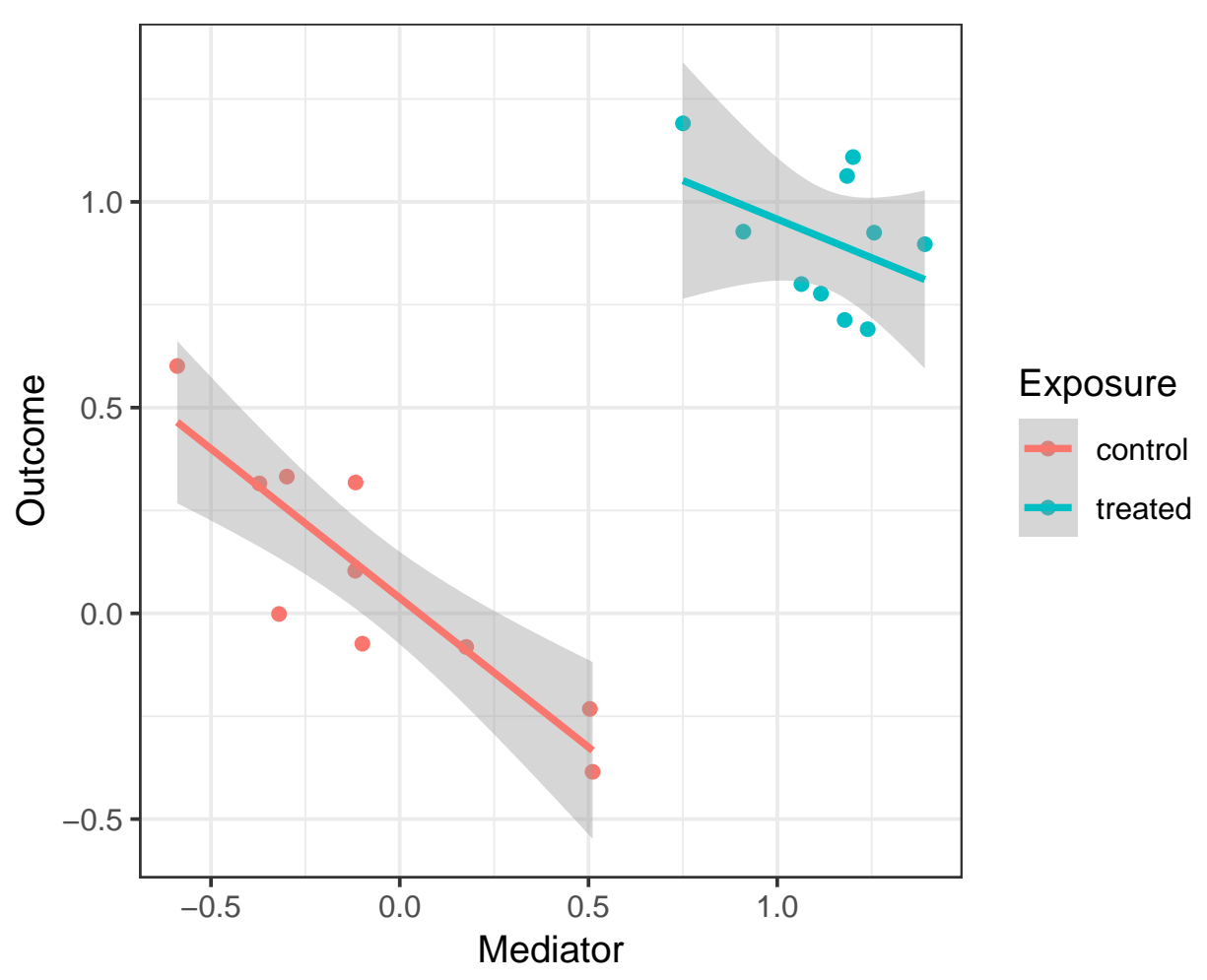

Figure 2: Inconsistent mediator.

challenging: $\theta_{2} \rightarrow \infty$, so $P\left(\hat{\theta}_{2}>0\right) \rightarrow 1$ and $p_{2} \rightarrow 0$. Under the null, we have $S=0$, and $S=0 \rightarrow \alpha_{1} \leq 0$, so we consider $\alpha_{1}=0$, because this is the most challenging value for Hitman.

The p-value from Hitman can be defined as a mixture for this challenging case:

$$
0.5 \max \left(p_{1}, p_{2}\right) \hat{S}+(1-\hat{S})\left(1-0.5 \max \left(p_{1}, p_{2}\right)\right)=0.5 p_{1} \hat{S}+(1-\hat{S})\left(1-0.5 p_{1}\right)
$$

To demonstrate this is a valid p-value, we need to show $P\left(p \leq u \mid H_{0}\right) \leq u$ for each $0 \leq u \leq 1$. We have that

$$
\begin{aligned}
& P\left(0.5 p_{1} \hat{S}+(1-\hat{S})\left(1-0.5 p_{1}\right) \leq u \mid \alpha_{1}=0, \hat{\theta}_{2}>>0, \hat{\lambda}_{1}>0\right) \\
= & \int_{a} P\left(0.5 p_{1} \hat{S}+(1-\hat{S})\left(1-0.5 p_{1}\right) \leq u \mid \hat{\alpha}_{1}=a, \hat{\theta}_{2}>>0, \hat{\lambda}_{1}>0\right) f\left(\hat{\alpha}_{1}=a \mid \alpha_{1}=0\right) d a
\end{aligned}
$$

where >> denotes much larger than; we leave $S=0$ out of equations 4 and 5 , because it is implied by the other two parameters; and equation 5 follows from equation 4 by the law of total probability. We have that $f\left(\hat{\alpha}_{1}=a \mid \alpha_{1}=0\right) \sim N\left(0, \sigma^{2}\right)$. Without loss of generality, we conveniently assume $\sigma=1$, so $f\left(\hat{\alpha}_{1}=a \mid \alpha_{1}=0\right)=\phi(a)$ and $\left(p_{1} \mid \hat{\alpha}_{1}=a\right)=2 \Phi(-|a|)$, because $\hat{\alpha}_{1}$ is tested with a two-sided test, where $\phi$ is the standard normal density and $\Phi$ is the standard normal cumulative distribution function. So, equation 5 simplifies to

$$
\begin{aligned}
& \int_{a} P\left(\Phi(-|a|) \hat{S}+(1-\hat{S})(1-\Phi(-|a|)) \leq u \mid \hat{\alpha}_{1}=a, \hat{\theta}_{2}>>0, \hat{\lambda}_{1}>0\right) \phi(a) d a \\
= & \int_{a \leq 0} P(1-\Phi(a) \leq u) \phi(a) d a+\int_{a>0} P(\Phi(-a) \leq u) \phi(a) d a
\end{aligned}
$$


Because if $a \leq 0$, then $\hat{S}=0$ and $\Phi(-|a|)=\Phi(a)$, whereas if $a>0$ then $\Phi(-|a|)=\Phi(-a)$.

We further simplify the second term in equation 6 :

$$
\begin{aligned}
\int_{a>0} P(\Phi(-a) \leq u) \phi(a) d a & =\int_{a>0} P\left(-a \leq \Phi^{-1}(u)\right) \phi(a) d a \\
& =\int_{a \leq 0} P\left(a \leq \Phi^{-1}(u)\right) \phi(-a) d a \\
& =\int_{a \leq 0} P\left(a \leq \Phi^{-1}(u)\right) \phi(a) d a \\
& =\int_{a \leq 0} \Phi\left(\Phi^{-1}(u)\right) \phi(a) d a \\
& =u \int_{a \leq 0} \phi(a) d a \\
& =u / 2
\end{aligned}
$$

We also simplify the first term in equation 6 :

$$
\begin{aligned}
\int_{a \leq 0} P(1-\Phi(a) \leq u) \phi(a) d a & =\int_{a \leq 0} P(-\Phi(a) \leq u-1) \phi(a) d a \\
& =\int_{a \leq 0} P(\Phi(a) \geq 1-u) \phi(a) d a \\
& =\int_{a \leq 0} P\left(a \geq \Phi^{-1}(1-u)\right) \phi(a) d a \\
& =\int_{a \leq 0} 1-\Phi\left(\Phi^{-1}(1-u)\right) \phi(a) d a \\
& =u \int_{a \leq 0} \phi(a) d a \\
& =u / 2
\end{aligned}
$$

Thus, $\forall u: 0 \leq u \leq 1, P\left(p \leq u \mid H_{0}\right) \leq u$, so Hitman controls its false positive rate.

\section{Simulations}

We validated our size and power following a simulation study (Barfield et al. 2017). This study's parameters were a subset of a previous simulation study (MacKinnon et al., 2002). Like (Barfield et al. 2017), we simulated data from $Y=t_{0}+t_{1} E+t_{2} M_{1}+t_{3} X+e_{Y}$, where $M_{1}$ defines the first mediator, which was simulated as $M_{1}=b_{0}+b_{1} E+b_{2} X+e_{M_{1}}$. This was the only mediator associated with exposure and outcome, and so was the only mediator whose p-value we examine.

Hitman relies on high-throughput data, so we simulated other mediators as $M_{i}=b_{0}+b_{2} X+e_{M_{i}}$ for $i \neq 1$, which are independent of the exposure and the outcome. (We index mediators using the subscript $i$, but suppress indices that indicate sample.) These other mediators are exploited by limma to estimate the shared technical variance, as would happen in an omics study.

$X$ and $E$ and the error terms $e_{Y}$ and $e_{M_{i}}$ for all $i$ were simulated as independent standard normal variables. We set $\theta_{0}=\theta_{3}=\beta_{0}=\beta_{2}=0.14$. Barfield et al. (2017) set the direct effect to be "small", $\theta_{1}=0.14$.

Hitman is only applicable when there is a robust overall effect, but these simulations include cases where there is no overall effect. Thus these simulations are not entirely appropriate for Hitman. However, we addressed this in part by setting the direct effect $\theta_{1}$ to be "large" $\left(\theta_{1}=0.59\right)$ as per (MacKinnon et al., 
2002). In this way, the simulations more closely match Hitman cases but can still be compared to previous simulations (Barfield et al., 2017; MacKinnon et al., 2002).

Like Barfield et al. (2017), we simulated all combinations of $\theta_{2}, \beta_{1} \in(0,0.14,0.39)$, which correspond to effects of "zero", "small," and "medium" size, respectively (MacKinnon et al., 2002). We tested in what proportion of 10,000 simulations $M_{1}$ of 100 mediators across 50 samples achieved a p-value $\leq 0.05$. Our results for these parameter values for Hitman, the joint significance test, and the mediate function from the $\mathrm{R}$ package mediation are shown in Table 1.

Table 1: Comparison of methods via simulation.

\begin{tabular}{rrrrr}
\hline$\beta_{1}$ & $\theta_{1}$ & Hitman & joint & mediate \\
\hline 0.00 & 0.00 & 0.006 & 0.002 & 0.002 \\
0.00 & 0.14 & 0.012 & 0.006 & 0.005 \\
0.14 & 0.00 & 0.016 & 0.008 & 0.007 \\
0.00 & 0.39 & 0.040 & 0.036 & 0.030 \\
0.39 & 0.00 & 0.048 & 0.038 & 0.028 \\
0.14 & 0.14 & 0.063 & 0.023 & 0.015 \\
0.14 & 0.39 & 0.206 & 0.108 & 0.103 \\
0.39 & 0.14 & 0.207 & 0.111 & 0.127 \\
0.39 & 0.39 & 0.682 & 0.539 & 0.574 \\
\hline
\end{tabular}

A statistical test's size is the probability of falsely rejecting the null hypothesis, which is the probability of a false positive or a Type 1 error. All the methods here control their size, as they maintain a false positive rate less than $5 \%$.

A statistical test's power is the probability that the test correctly rejects the null hypothesis when the alternative hypothesis is true, and it's inversely related to the probability of making a Type 2 error. To test Hitman's power, we look at the cases where both of $\theta_{2}$ and $\beta_{1}$ are positive. Here, Hitman's power is greater than the other methods.

\section{References}

Barfield R, Shen J, Just AC, Vokonas PS, Schwartz J, et al. 2017. Testing for the indirect effect under the null for genome-wide mediation analyses. Genet Epidemiol. 41(8):824-33

Benjamini Y, Hochberg Y. 1995. Controlling the false discovery rate: A practical and powerful approach to multiple testing. Journal of the Royal Statistical Society: Series B (Methodological). 57(1):289-300

Huang C-C, Tsai M-H, Wu Y-C, Chen K-T, Chuang H-W, et al. 2018. Activity dependent mammalian target of rapamycin pathway and brain derived neurotrophic factor release is required for the rapid antidepressant effects of puerarin. Am J Chin Med. 1-16

Judd CM, Kenny DA. 1981. Process analysis. Eval Rev. 5(5):602-19

Law CW, Chen Y, Shi W, Smyth GK. 2014. Voom: Precision weights unlock linear model analysis tools for RNA-seq read counts. Genome Biol. 15(2):R29

MacKinnon DP, Lockwood CM, Hoffman JM, West SG, Sheets V. 2002. A comparison of methods to test mediation and other intervening variable effects. Psychol Methods. 7(1):83-104

Pearl J. 2010. An introduction to causal inference. Int J Biostat. 6(2):Article 7

Pearl J. 2014a. Comment: Understanding simpsonparadox. The American Statistician. 68(1):8-13

Pearl J. 2014b. The deductive approach to causal inference. J Causal Inference. 2(2): 
bioRxiv preprint doi: https://doi.org/10.1101/817494; this version posted October 29, 2019. The copyright holder for this preprint (which was not certified by peer review) is the author/funder, who has granted bioRxiv a license to display the preprint in perpetuity. It is made available under aCC-BY 4.0 International license.

Ritchie ME, Phipson B, Wu D, Hu Y, Law CW, et al. 2015. Limma powers differential expression analyses for RNA-sequencing and microarray studies. Nucleic Acids Res. 43(7):e47 